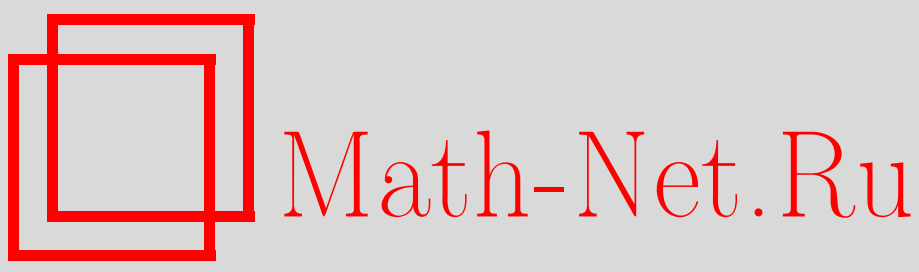

Вик. С. Куликов, Формула разложения на множители полного поворота с удвоенным числом нитей, Изв. РАН. Сер. матем., 2004, том 68, выпуск 1, 123-158

DOI: https://doi.org/10.4213/im468

Использование Общероссийского математического портала Math-Net.Ru подразумевает, что вы прочитали и согласны с пользовательским соглашением

http: //www.mathnet.ru/rus/agreement

Параметры загрузки:

IP : 3.85 .183 .62

26 апреля 2023 г., 17:58:49 


\title{
Формула разложения на множители полного поворота с удвоенным числом нитей
}

\begin{abstract}
Приведена формула для разложения на множители полного поворота в группе кос $\mathrm{Br}_{2 m}$, зависящая от четырех разложений на множители полного поворота в $\mathrm{Br}_{m}$. Используя эту формулу, мы строим симплектическое четырехмерное многообразие $X$ и два регулярно гомотопных общих накрытия $f_{i}: X \rightarrow \mathbb{C P}^{2}$ плоскости, разветвленные соответственно вдоль двух каспидальных кривых Гурвица $\bar{H}_{i} \subset \mathbb{C P}^{2}$ (без отрицательных ноудов), имеющих разные типы разложений на множители брэйд-монодромии. Описан класс фундаментальных групп дополнений к аффинным плоским кривым Гурвица в терминах образующих и определяющих соотношений.

Библиограффия: 17 наименований.
\end{abstract}

\section{Введение}

Пусть $(X, L)$ - поляризованная проективная поверхность над полем $\mathbb{C}$, где поляризация $L$ - это очень обильное линейное расслоение на $X$. Три общих сечения расслоения $L$ определяют обшее накрытие плоскости $f: X \rightarrow \mathbb{C P}^{2}$, разветвленное над алгебраической каспидальной кривой $\bar{H} \subset \mathbb{C P}^{2}$ (возможно, чтобы получить общее накрытие плоскости, вместо сечений расслоения $L$ необходимо взять три сечения расслоения $L^{\otimes 2}$ [8]). Недавно Д. Ору и Л. Кацарков (см. [2], [3]) получили следуюший результат в симплектическом случае. Пусть $(X, \omega)$ - компактное симплектическое четырехмерное многообразие с симплектической формой $\omega$, класс которой $[\omega]$ принадлежит $H^{2}(X, \mathbb{Z})$. Зафиксируем $\omega$-совместимую почти комплексную структуру $J$ и соответствующую риманову метрику $g$. Пусть $L-$ линейное расслоение на $X$ с первым классом Черна, равным $[\omega]$. Тогда при $k \gg 0$ линейное расслоение $L^{\otimes k}$ имеет достаточно много почти голоморфных сечений, так что можно выбрать из них три, дающие почти голоморфное общее накрытие $f_{k}: X \rightarrow \mathbb{C P}^{2}$ степени $N_{k}=k^{2} \omega^{2}$, разветвленное вдоль каспидальной кривой Гурвица $\bar{H}_{k}$ (понятие кривой Гурвица является обобщением понятия плоской алгебраической кривой; см. определение в $\S 5$ ). В алгебраическом случае если $\operatorname{deg} f \geqslant 12$, то $f$ определяется однозначно кривой $\bar{H}[10],[13]$. Любое общее накрытие $f: X \rightarrow \mathbb{C P}^{2}$ степени $N$, разветвленное вдоль каспидальной кривой Гурвища $\bar{H}$, определяет монодромию $\bar{\mu}$ (и определяется ею), т. е. эпиморфизм $\bar{\mu}: \pi_{1}\left(\mathbb{C P}^{2} \backslash \bar{H}\right) \rightarrow \mathfrak{S}_{N}$ в симметрическую группу $\mathfrak{S}_{N}$. Поэтому одной из основных задач является исследование свойств фундаментальных групп дополнений к кривым Гурвица с целью построить интересные примеры симплектических четырехмерных многообразий.

Работа частично поддержана фондами РФФИ (грант № 02-01-00786) и INTAS (грант № 00-0269). 
В работе [11] был определен класс $\mathscr{C}$ групп, названных $C$-группами. Этот класс совпадает (см. предложение 1.3) с классом, состоящим из групп, заданных конечным копредставлением следуюшего вида: для некоторого целого числа $m$, функции $h:\{1, \ldots, m\}^{2} \rightarrow \mathbb{Z}$ и подмножества

$$
W=\left\{w_{i, j, k} \in \mathbb{F}_{m} \mid 1 \leqslant i, j \leqslant m, 1 \leqslant k \leqslant h(i, j)\right\} \subset \mathbb{F}_{m}
$$

где $\mathbb{F}_{m}-$ свободная группа, порожденная алфавитом $\left\{x_{1}, \ldots, x_{m}\right\}$, группа $G \in \mathscr{C}$ задана копредставлением

$$
G_{W}=\left\langle x_{1}, \ldots, x_{m} \mid x_{i}=w_{i, j, k}^{-1} x_{j} w_{i, j, k}, w_{i, j, k} \in W\right\rangle .
$$

Пусть $\varphi_{W}: \mathbb{F}_{m} \rightarrow G_{W}-$ канонический эпиморфизм. Элементы $\varphi_{W}\left(x_{i}\right) \in G$, $1 \leqslant i \leqslant m$, и элементы, сопряженные им, называются $C$-образующими $C$-группы $G$. Пусть $f: G_{1} \rightarrow G_{2}$ - гомоморфизм $C$-групп. Он называется $C$-гомомор$\oint и з м о м$, если образы всех $C$-образуюших группы $G_{1}$ при гомоморфизме $f$ являются $C$-образуюшими $C$-группы $G_{2}$. Будем различать группы $G \in \mathscr{C}$ с точностью до $C$-изоморфизмов. Отметим, что класс $\mathscr{C}$ содержит подклассы $\mathscr{K}$ и $\mathscr{L}$ соответственно групп узлов и зацеплений, заданных с помошью копредставления Виртингера. В [11] было доказано, что класс $\mathscr{C}$ совпадает с классом фундаментальных групп дополнений к ориентируемым поверхностям в четырехмерной сфере $S^{4}$ (с обобщенным копредставлением Виртингера).

Доказывается (теорема 6.1 ), что класс $\mathscr{H}=\left\{\pi_{1}\left(\mathbb{C}^{2} \backslash H\right)\right\}$ фундаментальных групп дополнений к аффиинным кривым Гурвица $H$ (т.е. $H=\bar{H} \cap\left(\mathbb{C P}^{2} \backslash L_{\infty}\right)$, где $\bar{H}$ - кривая Гурвица в $\mathbb{C P}^{2}$ и $L_{\infty}-$ проективная прямая в $\mathbb{C P}^{2}$, находящаяся в общем положении относительно $\bar{H}$ ) совпадает с подклассом в $\mathscr{C}$, состояшим из $C$-групп $G_{W}$, заданных копредставлением (1) таким, что множество слов $\left\{\left[x_{i}, x_{1} \ldots x_{m}\right]\right\}_{i=1, \ldots, m}$ является подмножеством множества $W$ (через $[a, b]$ обозначен коммутант элементов $a$ и $b$ ). Мы также описываем (теорема 6.3) пересечение $\mathscr{H} \cap \mathscr{K}$ классов $\mathscr{H}$ и $\mathscr{K}$. Кроме того, доказывается (следствие 1.15), что класс $\mathscr{C}$ совпадает с классом групп $\mathscr{C}_{\mathrm{Br}}$, которые обладают копредставлением вида

$$
G_{B}=\left\langle x_{1}, \ldots, x_{m} \mid x_{i}^{-1} b\left(x_{i}\right)=\mathbf{1}, i=1, \ldots, m, b \in B\right\rangle
$$

для некоторого $m \in \mathbb{N}$ и конечно определенной подгруппы группы кос $\mathrm{Br}_{m}$, где $b\left(x_{i}\right)$ - образ элемента $x_{i}$ при стандартном действии элемента $b \in \mathrm{Br}_{m}$ на $\mathbb{F}_{m}$. Доказательство этих результатов базируется на формуле разложений на множители полного поворота в группе кос $\mathrm{Br}_{2 m}$ (см. $\left.\S 3\right)$. Эта формула также применяется в доказательстве существования симплектического четырехмерного многообразия $X$ и двух регулярно гомотопных общих накрытий $f_{i}: X \rightarrow \mathbb{C P}^{2}, i=1,2$, разветвленных соответственно над каспидальными кривыми Гурвица $\bar{H}_{i} \subset \mathbb{C P}^{2}$ (без отрицательных ноудов), имеющими разные типы разложений на множители брэйд-монодромии (см. теорему 7.7).

\section{§1. $C$-группы}

Пусть $\mathbb{F}$ - свободная группа, порожденная алфавитом $\left\{x_{j} \mid j \in \mathbb{N}\right\}$. Ниже через $\mathbb{F}_{m}$ обозначается подгруппа группы $\mathbb{F}$, порожденная элементами $x_{1}, \ldots, x_{m}$, и через $\mathbb{F}_{m, k}-$ подгруппа в $\mathbb{F}$, порожденная элементами $x_{k+1}, \ldots, x_{k+m}$. Обозна- 
чение $w\left(x_{1}, \ldots, x_{m}\right)$ будет использоваться для слов, состоящих из букв $x_{1}, \ldots, x_{m}$ и обратных к ним и рассматриваемых как элементы группы $\mathbb{F}_{m}$.

Пусть $W$ - некоторое подмножество в $\mathbb{F}_{m}$ и $N_{m}(W)$ - нормальное замыкание $W$ в $\mathbb{F}_{m}$. Обозначим через $\varphi_{W}: \mathbb{F}_{m} \rightarrow \mathbb{F}_{m} / N_{m}(W)=G(W)$ канонический эпиморфизм. Множество

$$
R_{W}=\left\{w_{j}\left(x_{1}, \ldots, x_{m}\right)=\mathbf{1} \mid w_{j} \in W\right\}
$$

будем называть множеством определяющих соотношений группы $G(W)$. Скажем, что соотношения $R_{W}$ влекут соотношение $\{w=\mathbf{1}\}$, если $w \in N_{m}(W)$. Иногда множество $R_{W}$ будем записывать в виде

$$
R_{W}=\left\{u_{j}\left(x_{1}, \ldots, x_{m}\right)=v_{j}\left(x_{1}, \ldots, x_{m}\right) \mid w_{j}(\bar{x})=u_{j}(\bar{x}) v_{j}^{-1}(\bar{x}) \in W\right\}
$$

для некоторого представления слов $w_{j} \in W$ как произведение $w_{j}=u_{j} v_{j}^{-1}$.

Пусть $W_{1} \subset \mathbb{F}_{m_{1}}, W_{2} \subset \mathbb{F}_{m_{2}}$ и $f: G\left(W_{1}\right) \rightarrow G\left(W_{2}\right)$ - гомоморфизм. Скажем, что $f$ является каноническим гомоморфизмом, если

$$
f\left(\varphi_{W_{1}}\left(x_{i}\right)\right)=\varphi_{W_{2}}\left(x_{i}\right)
$$

для всех $i \leqslant \min \left(m_{1}, m_{2}\right)$. В частности, если $W_{1} \subset W_{2}$ - два подмножества в $\mathbb{F}_{m}$, то существует канонический эпиморфизм

$$
\psi_{W_{1}, W_{2}}: G\left(W_{1}\right) \rightarrow G\left(W_{2}\right)=G\left(W_{1}\right) / N_{G\left(W_{1}\right)}\left(\varphi_{W_{1}}\left(W_{2} \backslash W_{1}\right)\right)
$$

где $N_{G\left(W_{1}\right)}\left(\varphi_{W_{1}}\left(W_{2} \backslash W_{1}\right)\right)$ - нормальное замыкание в группе $G\left(W_{1}\right)$ множества $\varphi_{W_{1}}\left(W_{2} \backslash W_{1}\right)$.

Скажем, что два множества соотношений

$$
R_{W_{i}}=\left\{w_{i, j}=\mathbf{1} \mid w_{i, j} \in W_{i}\right\}, \quad i=1,2,
$$

әквивалентны, если $N_{m}\left(W_{1}\right)=N_{m}\left(W_{2}\right)$. В общем случае для $k \leqslant m$ пусть $W_{1} \subset \mathbb{F}_{k}$ и $W_{2} \subset \mathbb{F}_{m}-$ два подмножества элементов из $\mathbb{F}_{k}$ и $\mathbb{F}_{m}$ соответственно. Множества соотношений

$$
\begin{aligned}
& R_{W_{1}}=\left\{w_{1, j}=\mathbf{1} \mid w_{1, j} \in W_{1}\right\}, \\
& R_{W_{2}}=\left\{w_{2, j}=\mathbf{1} \mid w_{2, j} \in W_{2}\right\}
\end{aligned}
$$

называются әквивалентными, если сушествует канонический изоморфизм $h$ : $G\left(W_{1}\right) \rightarrow G\left(W_{2}\right)$.

УТВЕРЖДЕНИЕ 1.1. Для $W_{1}, W_{2} \subset \mathbb{F}_{k} u \bar{W}_{1} \subset \mathbb{F}_{m}, m \geqslant k$, если множества соотношений $R_{W_{1}}$ и $R_{\bar{W}_{1}}$ являются эквивалентными, то множества соотношений $R_{W_{1} \cup W_{2}}$ и $R_{\bar{W}_{1} \cup W_{2}}$ әквивалентны.

ДоКАЗАТЕЛЬСТвО состоит из прямой проверки. 
УТВЕРЖДЕНИЕ 1.2. Пусть $w\left(x_{1}, \ldots, x_{m}\right)=x_{j_{1}}^{\varepsilon_{1}} \ldots x_{j_{n}}^{\varepsilon_{n}}$, где $\varepsilon_{k}= \pm 1, \quad k=$ $1, \ldots, n,-$ слово, состоящее из букв $x_{1}, \ldots, x_{m}$ и обратных $к$ ним, которое имеет буквенную длину $n \geqslant 2$. Тогда для любой пары $i_{1}, i_{2} \in\{1, \ldots, m\}$ соотношение $\left\{x_{i_{1}}=w^{-1} x_{i_{2}} w\right\}$ в $\mathbb{F}_{m}$ әквивалентно обгединению множеств соотношений

$$
\begin{aligned}
& R_{w}=\left\{x_{m+k}=x_{j_{k}}^{-\varepsilon_{k}} x_{m+k-1} x_{j_{k}}^{\varepsilon_{k}} \mid k\right.=2, \ldots, n-1\} \\
& \cup\left\{x_{m+1}=x_{j_{1}}^{-\varepsilon_{1}} x_{i_{2}} x_{j_{1}}^{\varepsilon_{1}}, x_{i_{1}}=x_{j_{n}}^{-\varepsilon_{n}} x_{m+n-1} x_{j_{n}}^{\varepsilon_{n}}\right\}
\end{aligned}
$$

в свободной гpуппе $\mathbb{F}_{m+n-1}$.

ДОКАЗАТЕЛЬСТвО состоит из прямой проверки.

Пусть $I$ - произвольное подмножество множества $\{1, \ldots, N\}^{3}$. Поскольку для $(i, j, k) \in\{1, \ldots, N\}^{3}$ соотношения $x_{i}=x_{j}^{-1} x_{k} x_{j}$ и $x_{k}=x_{j} x_{i} x_{j}^{-1}$ эквивалентны, утверждения 1.1 и 1.2 влекут следующее предложение.

ПРЕДЛОжЕНИЕ 1.3. Каждая $C$-группа $G$ канонически $C$-изоморфна $C$-группе $\bar{G}$, заданной $C$-копредставлением вида

$$
\bar{G} \simeq\left\langle x_{1}, \ldots, x_{N} \mid x_{i}=x_{j}^{-1} x_{k} x_{j},(i, j, k) \in I\right\rangle,
$$

m.е. определение C-групп, данное во введении, совпадает с определением C-групп, данным в [11].

Отметим, что соотношение $x_{i}=x_{j}$ является $C$-соотношением, так как оно может быть записано в виде $x_{i}=x_{j}^{-1} x_{j} x_{j}$.

ПримеР. Пусть $\mathrm{Br}_{m}$ - группа кос с $m$ нитями. Как известно, она порождается образуюшими $a_{1}, \ldots, a_{m-1}$, связанными соотношениями

$$
\begin{gathered}
a_{i} a_{i+1} a_{i}=a_{i+1} a_{i} a_{i+1}, \quad 1 \leqslant i \leqslant m-1, \\
a_{i} a_{k}=a_{k} a_{i}, \quad|i-k| \geqslant 2 .
\end{gathered}
$$

Группа кос $\mathrm{Br}_{m}$, заданная копредставлением (3), обладает естественной структурой $C$-группы. Действительно, пусть

$$
\begin{aligned}
R_{W\left(\mathrm{Br}_{m}\right)}= & \left\{x_{2 i+1}=x_{2 i-1}^{-1} x_{2 i} x_{2 i-1} \mid i=1, \ldots, m-1\right\} \\
& \cup\left\{x_{2 i-1}=x_{2 i}^{-1} x_{2 i+1} x_{2 i} \mid i=1, \ldots, m-1\right\} \\
& \cup\left\{x_{2 i-1}=x_{2 j-1}^{-1} x_{2 i-1} x_{2 j-1}|| i-j \mid \geqslant 2,1 \leqslant i, j \leqslant m-1\right\}
\end{aligned}
$$

- множество $C$-соотношений в $\mathbb{F}_{2 m-3}$. Положим $G_{\mathrm{Br}_{m}}=G\left(W\left(\mathrm{Br}_{m}\right)\right)$. Можно проверить, что гомоморфизм $f: \mathrm{Br}_{m} \rightarrow G_{\mathrm{Br}_{m}}$, заданньй на образующих следуюшим образом: $f\left(a_{i}\right)=\varphi_{W}\left(x_{2 i-1}\right), i=1, \ldots, m-1$, является изоморфизмом.

Для множества $W=W_{f}=\left\{w_{j}\left(x_{1}, \ldots, x_{m}\right) \in \mathbb{F}_{m} \mid j \in J\right\}$, где $f: J \rightarrow \mathbb{F}_{m}-$ отображение из множества $J$ и образ $f(j)$ элемента $j \in J$ обозначен через $w_{j}$, множество

$$
\operatorname{sh}(W)=\operatorname{sh}_{m}(W)=\left\{w_{j}\left(x_{m+1}, \ldots, x_{2 m}\right) \mid j \in J\right\}
$$


назьвается сдвигом множества $W$ на $m$. Положим

$$
W_{\bmod m}=\left\{x_{i}^{-1} x_{m+i} \mid i=1, \ldots, m\right\}
$$

и назовем множества

$$
\begin{gathered}
d(W)=d_{m}(W)=W \cup \operatorname{sh}(W) \cup W_{\bmod m} \\
\bar{d}(W)=\bar{d}_{m}(W)=\operatorname{sh}(W) \cup W_{\bmod m}
\end{gathered}
$$

в $\mathbb{F}_{2 m}$ соответственно удвоением и приведенным удвоением множества $W$.

Удвоение (соответственно, приведенное удвоение) множества $W$ может быть итерировано, если положить

$$
d^{n}(W)=d_{2^{n-1} m}\left(d^{n-1}(W)\right) \in \mathbb{F}_{2^{n} m}
$$

УТВЕРЖДЕНИЕ 1.4. Для любого $n \in \mathbb{N}$ множсества соотношений $R_{W}$, $R_{d^{n}(W)}$ и $R_{\bar{d}^{n}(W)}$ әквивалентны.

ДоКАЗАТЕЛЬСТВо состоит из прямой проверки.

Пусть $B$ - подгруппа в $\operatorname{Aut}\left(\mathbb{F}_{m}\right)$. Обозначим через $R_{W\left(B, \mathbb{F}_{m}\right)}$ множество соотношений:

$$
\left.R_{W\left(B, \mathbb{F}_{m}\right.}\right)=\left\{w g(w)^{-1}=\mathbf{1} \mid w \in \mathbb{F}_{m}, g \in B\right\} .
$$

УТВЕРЖДЕНИЕ 1.5. Пусть $y_{1}, \ldots, y_{m} \in \mathbb{F}_{m}$ - әлементы, порождающие группу $\mathbb{F}_{m}$. Тогда для подгруппь $B \subset \operatorname{Aut}\left(\mathbb{F}_{m}\right)$ множество соотношений $R_{W(\text { В, }}$ ) эквивалентно мнохсеству

$$
R_{W(B, \bar{y})}=\left\{y_{i} g\left(y_{i}\right)^{-1}=\mathbf{1} \mid 1 \leqslant i \leqslant m, g \in B\right\}
$$

ДокАЗАТЕльство. Заметим, что для доказательства утверждения достаточно показать, что если элементы $w_{1} g\left(w_{1}\right)^{-1}$ и $w_{2} g\left(w_{2}\right)^{-1}$ принадлежат группе $N_{m}(W(B, \bar{y}))$ для некоторых $w_{1}, w_{2} \in \mathbb{F}_{m}$, то

$$
\begin{gathered}
w_{1}^{-1} g\left(w_{1}^{-1}\right)^{-1} \in N_{m}(W(B, \bar{y})), \\
w_{1} w_{2} g\left(w_{1} w_{2}\right)^{-1} \in N_{m}(W(B, \bar{y})) .
\end{gathered}
$$

Если $w_{1} g\left(w_{1}\right)^{-1} \in N_{m}(W(B, \bar{y}))$, то

$$
\left(w_{1} g\left(w_{1}\right)^{-1}\right)^{-1}=g\left(w_{1}\right) w_{1}^{-1} \in N_{m}(W(B, \bar{y}))
$$

и, следовательно,

$$
w_{1}^{-1} g\left(w_{1}^{-1}\right)^{-1}=w_{1}^{-1}\left(g\left(w_{1}\right) w_{1}^{-1}\right) w_{1} \in N_{m}(W(B, \bar{y})) .
$$

Если $w_{1} g\left(w_{1}\right)^{-1}, w_{2} g\left(w_{2}\right)^{-1} \in N_{m}(W(B, \bar{y}))$, то

$$
\begin{aligned}
w_{1} w_{2} g\left(w_{1} w_{2}\right)^{-1} & =w_{1} w_{2} g\left(w_{2}\right)^{-1} g\left(w_{1}\right)^{-1} \\
& =w_{1}\left(w_{2} g\left(w_{2}\right)^{-1}\right) w_{1}^{-1}\left(w_{1} g\left(w_{1}\right)^{-1}\right) \in N_{m}(W(B, \bar{y})) .
\end{aligned}
$$


УТВеРЖДДЕНИЕ 1.6. Если подгруппа $B \subset \operatorname{Aut}\left(\mathbb{F}_{m}\right)$ порождается әлементами $b_{1}, \ldots, b_{n}$, а әлементы $y_{1}, \ldots, y_{m}$ порождают $\mathbb{F}_{m}$, то множество соотношений $R_{W\left(B, \mathbb{F}_{m}\right)}$ әквивалентно множеству

$$
R_{W(\bar{b}, \bar{y})}=\left\{y_{i} b_{j}\left(y_{i}\right)^{-1}=\mathbf{1} \mid 1 \leqslant i \leqslant m, 1 \leqslant j \leqslant n\right\} .
$$

ДокАЗАТЕЛЬСТво. Согласно утверждению 1.5 достаточно показать, что если элементы $w g_{1}(w)^{-1}$ и $w g_{2}(w)^{-1}$ принадлежат группе $N_{m}(W(\bar{b}, \bar{y}))$ для некоторых $g_{1}, g_{2} \in B$ и всех $w \in \mathbb{F}_{m}$, то $w\left(g_{1}^{-1}(w)\right)^{-1} \in N_{m}(W(\bar{b}, \bar{y}))$ и $w\left(g_{1} g_{2}(w)\right)^{-1} \in$ $N_{m}(W(\bar{b}, \bar{y}))$.

Если $w g_{1}(w)^{-1} \in N_{m}(W(\bar{b}, \bar{y}))$ для всех $w \in \mathbb{F}_{m}$, то

$$
\left(w\left(g_{1}^{-1}(w)\right)^{-1}\right)^{-1}=\left(g_{1}^{-1}(w)\right) w^{-1}=\left(g_{1}^{-1}(w)\right)\left(g_{1}\left(g_{1}^{-1}(w)\right)\right)^{-1} \in N_{m}(W(\bar{b}, \bar{y})) .
$$

Если $w\left(g_{1}(w)\right)^{-1}, w\left(g_{2}(w)\right)^{-1} \in N_{m}(W(\bar{b}, \bar{y}))$ для всех $w \in \mathbb{F}_{m}$, то

$$
w g_{1} g_{2}(w)^{-1}=w g_{1}\left(g_{2}(w)\right)^{-1}=\left(w g_{2}(w)^{-1}\right)\left(g_{2}(w) g_{1}\left(g_{2}(w)\right)^{-1}\right) \in N_{m}(W(\bar{b}, \bar{y})) .
$$

Группа кос $\mathrm{Br}_{m}$ с $m$ нитями действует на $\mathbb{F}_{m}$. Далее мы зафиксируем множество $\left\{a_{1}, \ldots, a_{m-1}\right\}$ так называемых стандартных образуюших, т. е. образуюших группы $\mathrm{Br}_{m}$, связанных соотношениями

$$
\begin{gathered}
a_{i} a_{i+1} a_{i}=a_{i+1} a_{i} a_{i+1}, \quad 1 \leqslant i \leqslant n-1, \\
a_{i} a_{k}=a_{k} a_{i}, \quad|i-k| \geqslant 2,
\end{gathered}
$$

и действующих на $\mathbb{F}_{m}$ следуюшим образом:

$$
\begin{gathered}
a_{i}\left(x_{j}\right)=x_{j}, \quad \text { если } \quad i \neq j, j+1, \\
a_{i}\left(x_{i}\right)=x_{i} x_{i+1} x_{i}^{-1}, \quad a_{i}\left(x_{i+1}\right)=x_{i} .
\end{gathered}
$$

Следовательно, каждой подгруппе $B \subset \mathrm{Br}_{m}$ можно сопоставить множество соотношений $R_{W\left(B, \mathbb{F}_{m}\right)}$. Как и в случае свободных групп, при $k \leqslant m$ группа $\mathrm{Br}_{k}$ будет отождествляться с подгруппой группы $\mathrm{Br}_{m}$, порожденной первыми $k-1$ стандартными образуюшими $a_{1}, \ldots, a_{k-1}$, и через $B_{k, i}, k+i \leqslant m$, будет обозначаться подгруппа групшы $\mathrm{Br}_{m}$, порожденная элементами $a_{i+1}, \ldots, a_{i+k-1}$. В частности, $B_{k, 0}=\mathrm{Br}_{k}$. Пусть $\Delta_{k, i}-$ элемент Гарсайда в группе кос $B_{k, i}$ :

$$
\Delta_{k, i}=\left(a_{i+1} \ldots a_{i+k-1}\right) \ldots\left(a_{i+1} a_{i+2} a_{i+3}\right)\left(a_{i+1} a_{i+2}\right) a_{i+1} .
$$

Имеем

$$
\Delta_{k, i}\left(x_{i+j}\right)=\left(x_{i+1} \ldots x_{i+k-j}\right) x_{i+k-j+1}\left(x_{i+1} \ldots x_{i+k-j}\right)^{-1}
$$

для $i+1 \leqslant j \leqslant i+k$ (по определению $x_{i+1} \ldots x_{i+k-j}=\mathbf{1}$, если $j=i+k$ ) и

$$
\Delta_{k}\left(x_{j}\right)=x_{j}
$$


для $j \leqslant i$ и $j>i+k$. Элемент Гарсайда в группе кос $\mathrm{Br}_{k}$ будет обозначаться через $\Delta_{k}$.

Обозначим через $\mathrm{sh}=\mathrm{sh}_{m}: \mathrm{Br}_{m} \rightarrow \mathrm{Br}_{2 m}$ гомоморфизм, заданный на образуюших следуюшим образом: $\operatorname{sh}\left(a_{j}\right)=a_{m+j}$ для $j=1, \ldots, m-1$. Пусть $B-$

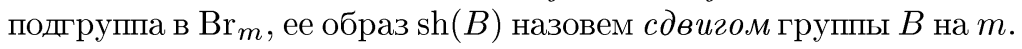

$\mathrm{B}$ дальнейшем группа $\mathbb{F}_{k}$ будет отождествляться с фундаментальной группой $\pi_{1}\left(D_{k} \backslash\left\{p_{1}, \ldots, p_{k}\right\}, p_{0}\right)$, где $D_{k}=\{z \in \mathbb{C}|| z \mid \leqslant k+1\}, p_{0}=-i(k+1)$, $p_{j}=-j, j=1, \ldots, k$, и для каждого $j$ элемент $x_{j}$ будет отождествляться с петлей, состоящей из пути вдоль отрезка

$$
\gamma_{j}=\{z \in \mathbb{C} \mid z=-i(k+1)-t(j+i(\varepsilon-k-1)), 0 \leqslant t \leqslant 1\}
$$

для некоторого $\varepsilon, 0<\varepsilon \ll 1$, обхода против часовой стрелки вдоль окружности $\{|z+j|=\varepsilon\}$ и возврата в точку $p_{0}$ вдоль $l_{j}$ (см. рис. 1$)$. Для выбранного таким образом базиса $x_{1}, \ldots, x_{k}$ стандартные образующие $a_{j}$ группы $\mathrm{Br}_{k}$, $j=1, \ldots, k-1$, отождествляются с полуповоротами, определяемыми сегментами $[-j-1,-j]=\{z=-(j+1)+t \mid 0 \leqslant t \leqslant 1\}$ (см., например, [17]). Элемент $l_{k}=x_{1} \ldots x_{k}$ в $\pi_{1}\left(D_{k} \backslash\left\{p_{1}, \ldots, p_{k}\right\}, p_{0}\right)$ представляется обходом против часовой стрелки вдоль границы $\partial D_{k}$ диска $D_{k}$ и является неподвижным элементом действия группы $\mathrm{Br}_{k}$.

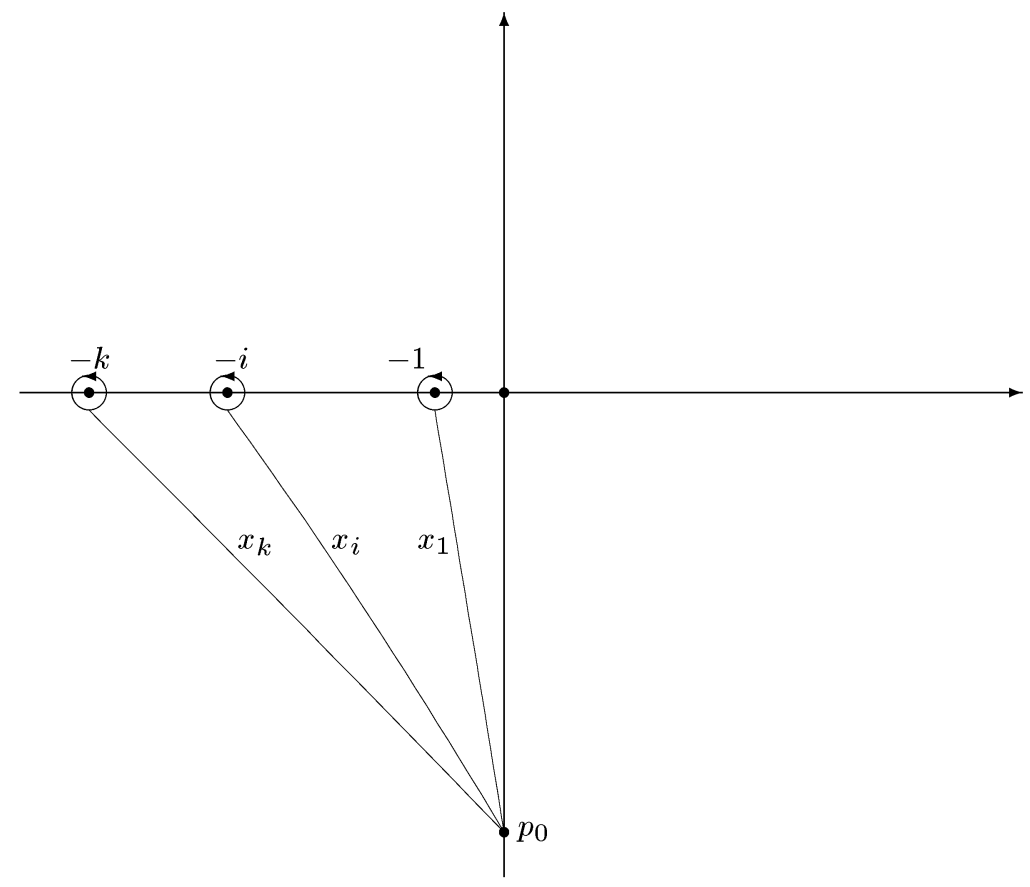

Рис. 1 
Скажем, что упорядоченное множество $\left\{y_{1}, \ldots, y_{m}\right\}$ элементов из $\mathbb{F}_{m}$ является хорошим геометрическим базисом группы $\mathbb{F}_{m}$, если найдется элемент $b \in \mathrm{Br}_{m}$ такой, что $y_{j}=b\left(x_{j}\right)$ для $j=1, \ldots, m$. Упорядоченное множество $\left\{y_{1}, \ldots, y_{k}\right\}$, $k \leqslant m$, элементов из $\mathbb{F}_{m}$ называется хорошим, если множество $\left\{y_{1}, \ldots, y_{k}\right\}$ может быть расширено до хорошего геометрического базиса $\left\{y_{1}, \ldots, y_{m}\right\}$ групшы $\mathbb{F}_{m}$. Известно, что $\left\{y_{1}, \ldots, y_{m}\right\}$ является хорошим геометрическим базисом в $\mathbb{F}_{m}$ тогда и только тогда, когда каждый элемент $y_{j}$ сопряжен с некоторым $x_{k(j)}$ в $\mathbb{F}_{m}$ и $y_{1} \ldots y_{m}=l_{m}$.

Рассмотрим элемент $w\left(x_{1}, \ldots, x_{m}\right)=x_{j_{1}}^{\varepsilon_{1}} \ldots x_{j_{n}}^{\varepsilon_{n}}, \varepsilon_{j}= \pm 1$, в $\mathbb{F}_{m}$ буквенной длины $n$. Для $k>n$ и $p \geqslant 1$ элемент

$$
\mathrm{m}_{p m k, k, m}(w)=x_{\left(p k-\varepsilon_{1}(k-1)\right) m+j_{1}}^{\varepsilon_{1}} \ldots x_{\left(p k-\varepsilon_{n}(k-n)\right) m+j_{n}}^{\varepsilon_{n}} \in \mathbb{F}_{m(p+1) k}
$$

называется раздвижкой элемента $w$ на $m$ с центром в $p m k$.

Далее нам понадобится следующее утверждение, являющееся очевидным с геометрической точки зрения.

УТВЕРЖДЕНИЕ 1.7. Пусть $w_{k}\left(x_{1}, \ldots, x_{m}\right)=x_{j_{k, 1}}^{\varepsilon_{k, 1}} \ldots x_{j_{k, n_{k}}}^{\varepsilon_{k, n_{k}}}, \varepsilon_{k, j}= \pm 1$, $k=1,2,-$ два слова, состоящие из букв $x_{1}, \ldots, x_{m}$ и обратных $к$ ним и имеющие буквенные длины $n_{k}$, меньшие чем $n$. Тогда для каждой пары чисел $i_{1}, i_{2}$ такой, что $1 \leqslant i_{1}, i_{2} \leqslant m$, пара әлементов $y_{1}, y_{2}$, где $y_{1}=\bar{w}_{1} x_{n m+i_{1}} \bar{w}_{1}^{-1}$, $y_{2}=\bar{w}_{2} x_{3 n m+i_{2}} \bar{w}_{2}^{-1}$, является хорошей в $\mathbb{F}_{4 m n}$, әде

$$
\begin{aligned}
\bar{w}_{1}=\mathrm{m}_{m n, n, m}\left(w_{1}\right) & =x_{\left(n-\varepsilon_{1,1}(n-1)\right) m+j_{1,1}}^{\varepsilon_{1,1}} \ldots x_{\left(n-\varepsilon_{1, n_{1}}\left(n-n_{1}\right)\right) m+j_{1, n_{1}}}^{\varepsilon_{1, n_{1}}}, \\
\bar{w}_{2}=\mathrm{m}_{3 m n, n, m}\left(w_{2}\right) & =x_{\left(3 n-\varepsilon_{2,1}(n-1)\right) m+j_{2,1}}^{\varepsilon_{2,1}} \ldots x_{\left(3 n-\varepsilon_{2, n_{2}}\left(n-n_{2}\right)\right) m+j_{2, n_{2}}}^{\varepsilon_{2, n_{2}}} .
\end{aligned}
$$

ДокАЗАТЕльство. Пара элементов $y_{1}$ и $y_{2}$ представляется парой петель вида, аналогичного тому, который изображен на рис. 2. Очевидно, такие пары $y_{1}, y_{2}$ являются хорошими.

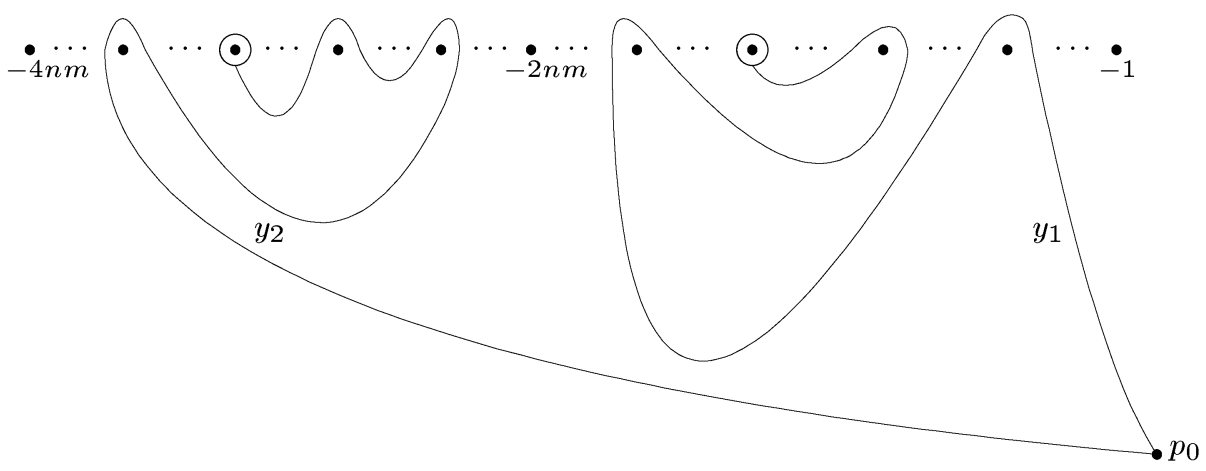

Рис. 2 
Пусть $h:\{1, \ldots, m\}^{2} \rightarrow \mathbb{Z}$ - неотрицательная целочисленная функция. Положим

$$
I_{h}=\left\{(i, j, k) \in \mathbb{Z}^{3} \mid 1 \leqslant i, j \leqslant m, 1 \leqslant k \leqslant h(i, j)\right\},
$$

и пусть $f: I_{h} \rightarrow \mathbb{F}_{m}$ - отображение в $\mathbb{F}_{m}$.

УТВЕРЖДЕНИЕ 1.8. Для любого отображсения $f: I_{h} \rightarrow \mathbb{F}_{m}$ существуют число $M \in \mathbb{N}$, конечное множество $I_{h, f} \subset\{1, \ldots, M\}^{2}$ и отображсние $F: I_{h, f} \rightarrow \mathbb{F}_{M}^{2}=\mathbb{F}_{M} \times \mathbb{F}_{M}$ такие, что:

(i) пара $F(i, j)=\left(y_{i}, y_{j}\right)$ является хорошей в $\mathbb{F}_{M}$ для кажсдого $(i, j) \in I_{h, f}$;

(ii) множества соотношений

$$
\begin{gathered}
R_{W_{f}}=\left\{x_{i}^{-1} w_{i, j, k} x_{j} w_{i, j, k}^{-1}=\mathbf{1} \mid(i, j, k) \in I_{h}, w_{i, j, k}=f(i, j, k)\right\}, \\
R_{W_{F}}=\left\{y_{i}^{-1} y_{j}=\mathbf{1} \mid\left(y_{i}, y_{j}\right) \in \operatorname{Im} F\right\}
\end{gathered}
$$

являются әквивалентными.

ДокАЗАТЕльство. Согласно утверждению 1.7 существует целое число $N$ такое, что пары

$$
\left(x_{i}, \mathrm{~m}_{N m, N, m}\left(w_{i, j, k}\right) x_{N m+j} \mathrm{~m}_{N m, N, m}\left(w_{i, j, k}\right)^{-1}\right)
$$

являются хорошими для всех $(i, j, k) \in I_{h}$. Очевидно, множество соотношений $R_{W_{f}}$ в $\mathbb{F}_{m}$ эквивалентно объединению двух множеств соотношений $R_{\mathrm{m}\left(W_{f}\right)}$ и $R_{\bmod m}$ в $\mathbb{F}_{2 N m}$, где

$$
\begin{gathered}
R_{\mathrm{m}\left(W_{f}\right)}=\left\{x_{i}=\mathrm{m}_{N m, N, m}\left(w_{i, j, k}\right) x_{N m+j} \mathrm{~m}_{N m, N, m}\left(w_{i, j, k}\right)^{-1} \mid(i, j, k) \in I_{h}\right\}, \\
R_{\bmod m}=\left\{x_{i}=x_{j} \mid i \equiv j \bmod m\right\} .
\end{gathered}
$$

Для каждого $i \leqslant m$ и каждого $k>0$ пара $x_{i}, x_{i+m k}$ является хорошей и, следовательно, утверждение будет доказано, если положить $M=2 N m$ и $R_{W_{F}}=$ $R_{\mathrm{m}\left(W_{f}\right)} \cup R_{\bmod m}$

В соответствии с утверждением 1.6 для подгруппы $B$ группы $\mathrm{Br}_{m}$, порожденной элементами $b_{1}, \ldots, b_{n}$, группа $G\left(W\left(B, \mathbb{F}_{m}\right)\right)$ канонически изоморфна групше

$$
G(W(\bar{b}, \bar{x}))=\left\langle x_{1}, \ldots, x_{m} \mid x_{i}^{-1} b_{j}\left(x_{i}\right)=\mathbf{1}, i=1, \ldots, m, j=1, \ldots, n\right\rangle .
$$

УТВЕРЖДЕНИЕ 1.9. Копредставление (8) определяет на $G(W(\bar{b}, \bar{x}))$ струкmypy $C$-грynnbl.

ДокаЗАТеЛЬСтво. Имеем

$$
b\left(x_{i}\right)=w_{b, i}^{-1}(\bar{x}) x_{j} w_{b, i}(\bar{x})
$$

для некоторого $j=\sigma_{b}(i)$ и некоторого $w_{b, i}(\bar{x}) \in \mathbb{F}_{m}$, где $\sigma_{b} \in \mathfrak{S}_{m}$ - образ элемента $b \in \mathrm{Br}_{m}$ при каноническом эпиморфизме $\sigma: \mathrm{Br}_{m} \rightarrow \mathfrak{S}_{m}$ с ядром $\operatorname{ker} \sigma=P_{m}$ (через $\mathfrak{S}_{m}$ обозначена симметрическая группа, действующая на множестве $\{1, \ldots, m\}$, и через $P_{m}$ - группа крашеных кос). Поэтому соотношения $x_{i}^{-1} b_{j}\left(x_{i}\right)=\mathbf{1}$ совпадают с $C$-соотношениями $x_{i}=w_{b_{j}, i}^{-1}(\bar{x}) x_{j} w_{b_{j}, i}(\bar{x})$.

Следуюшая лемма является хорошо известной. 
ЛЕмма 1.10. Пусть $b_{k}=g a_{j}^{k+1} g^{-1} \in \mathrm{Br}_{m}$. Тогда для кажсдого $k=0,1,2,-3$ множество соотношений

$$
\left\{x_{i}^{-1} b_{k}\left(x_{i}\right)=\mathbf{1} \mid i=1, \ldots, m\right\}
$$

в $\mathbb{F}_{m}$ әквивалентно соответственно одному соотношению:

(0) $g\left(x_{j}\right)=g\left(x_{j+1}\right)$, если $k=0$;

(1) коммутант $\left[g\left(x_{j}\right), g\left(x_{j+1}\right)\right]=\mathbf{1}$, если $k=1$;

(2) $g\left(x_{j}\right) g\left(x_{j+1}\right) g\left(x_{j}\right)=g\left(x_{j+1}\right) g\left(x_{j}\right) g\left(x_{j+1}\right)$, если $k=2$;

$(-3)$ коммутант $\left[g\left(x_{j}\right), g\left(x_{j+1}\right)\right]=1$, если $k=-3$.

ДокАЗАТЕльство. Рассмотрим свободный базис $\left\{y_{1}, \ldots, y_{m}\right\}$ группы $\mathbb{F}_{m}$, где $y_{i}=g\left(x_{i}\right)$ для $i=1, \ldots, m$. Согласно утверждению 1.6 множества соотношений $\left\{x_{i}^{-1} b_{k}\left(x_{i}\right)=\mathbf{1} \mid i=1, \ldots, m\right\}$ и $\left\{y_{i}^{-1} b_{k}\left(y_{i}\right)=\mathbf{1} \mid i=1, \ldots, m\right\}$ являются эквивалентными для каждого $k$.

Имеем

$$
b_{k}\left(y_{i}\right)=g\left(x_{i}\right)=g a_{j}^{k+1} g^{-1}\left(g\left(x_{i}\right)\right)=g\left(x_{i}\right)=y_{i},
$$

если $i \neq j, j+1$,

$$
\begin{aligned}
b_{k}\left(y_{j}\right) & =g a_{j}^{k+1}\left(x_{j}\right) \\
& = \begin{cases}g\left(x_{j} x_{j+1} x_{j}^{-1}\right), & \text { если } k=0, \\
g\left(\left(x_{j} x_{j+1}\right) x_{j}\left(x_{j} x_{j+1}\right)^{-1}\right), & \text { если } k=1, \\
g\left(\left(x_{j} x_{j+1} x_{j}\right) x_{j+1}\left(x_{j} x_{j+1} x_{j}\right)^{-1}\right), & \text { если } k=2,\end{cases}
\end{aligned}
$$

И

$$
\begin{aligned}
b_{k}\left(y_{j+1}\right) & =g a_{j}^{k+1}\left(x_{j+1}\right) \\
& = \begin{cases}g\left(x_{j}\right), & \text { если } k=0, \\
g\left(x_{j} x_{j+1} x_{j}^{-1}\right), & \text { если } k=1, \\
g\left(\left(x_{j} x_{j+1}\right) x_{j}\left(x_{j+1} x_{j}\right)^{-1}\right), & \text { если } k=2 .\end{cases}
\end{aligned}
$$

Следовательно,

$$
b_{k}\left(y_{j}\right)= \begin{cases}y_{j} y_{j+1} y_{j}^{-1}, & \text { если } k=0, \\ \left(y_{j} y_{j+1}\right) y_{j}\left(y_{j} y_{j+1}\right)^{-1}, & \text { если } k=1, \\ \left(y_{j} y_{j+1} y_{j}\right) y_{j+1}\left(y_{j} y_{j+1} y_{j}\right)^{-1}, & \text { если } k=2,\end{cases}
$$

и

$$
b_{k}\left(y_{j+1}\right)= \begin{cases}y_{j}, & \text { если } k=0, \\ y_{j} y_{j+1} y_{j}^{-1}, & \text { если } k=1, \\ \left(y_{j} y_{j+1}\right) y_{j}\left(y_{j+1} y_{j}\right)^{-1}, & \text { если } k=2 .\end{cases}
$$

Таким образом, для каждого $k$ множество соотношений $\left\{y_{i}^{-1} b_{k}\left(y_{i}\right)=\mathbf{1} \mid i=\right.$ $1, \ldots, m\}$ сводится к единственному из следуюших соотношений:

$$
\begin{aligned}
y_{j} y_{j+1}^{-1} & =\mathbf{1}, & & \text { если } k=0, \\
y_{j} y_{j+1} y_{j}^{-1} y_{j+1}^{-1} & =\mathbf{1}, & & \text { если } k=1, \\
\left(y_{j} y_{j+1} y_{j}\right)\left(y_{j+1} y_{j} y_{j+1}\right)^{-1} & =\mathbf{1}, & & \text { если } k=2 .
\end{aligned}
$$


УТВЕРЖДЕНИЕ 1.11. Пусть $y_{1}, y_{2}$ - хорошая пара в $\mathbb{F}_{m}$. Тогда существует такой әлемент $b=g a_{1} g^{-1} \in \mathrm{Br}_{m}$, что множество соотношений $R_{W\left(\langle b\rangle, \mathbb{F}_{m}\right)}$ әквивалентно соотношению

$$
R_{b}=\left\{y_{1}^{-1} y_{2}=\mathbf{1}\right\}
$$

ДокАЗАТЕльство. Поскольку $y_{1}, y_{2}$ - хорошая пара, она может быть включена в хороший геометрический базис $\left\{y_{1}, \ldots, y_{m}\right\}$ группы $\mathbb{F}_{m}$. Следовательно, найдется элемент $g \in \mathrm{Br}_{m}$ такой, что $g\left(x_{i}\right)=y_{i}$ для $i=1, \ldots, m$. Согласно лемме $1.10 R_{W\left(\langle b\rangle, \mathbb{F}_{m}\right)}$ эквивалентно соотношению $R_{b}$.

Рассмотрим группу кос $\mathrm{Br}_{2 m}$. Положим $a_{m, i}=a_{i} a_{2 m-i}$,

$$
\bar{c}_{m, i}=\left(a_{m, i} a_{m, i+1} \ldots a_{m, m-1}\right) a_{m}\left(a_{m, i} a_{m, i+1} \ldots a_{m, m-1}\right)^{-1}
$$

для $1 \leqslant i \leqslant m-1, \bar{c}_{m, m}=a_{m}$ и

$$
c_{m, i}=\Delta_{m, m}^{-1} \bar{c}_{m, i} \Delta_{m, m}
$$

УТВЕРЖДЕНИЕ 1.12. Множество соотношений $R_{W\left(\left\langle c_{m, i}\right\rangle, \mathbb{F}_{2 m}\right)}$ әквивалентно соотношению

$$
R_{c_{m, i}}=\left\{x_{m+i}^{-1} x_{i}=\mathbf{1}\right\}
$$

ДоКАЗАТЕЛЬСТво. Используя равенство (7), можно проверить, что

$$
\Delta_{m, m}^{-1}\left(a_{m, i} a_{m, i+1} \ldots a_{m, m-1}\right)\left(x_{m}\right)=x_{i}
$$

и

$$
\Delta_{m, m}^{-1}\left(a_{m, i} a_{m, i+1} \ldots a_{m, m-1}\right)\left(x_{m+1}\right)=x_{m+i}
$$

Следовательно, утверждение следует из (10), (11) и утверждения 1.10.

Для каждой подгруппы $B$ группы кос $\mathrm{Br}_{m}$ обозначим через $d(B)=d_{m}(B)$ (соответственно, $\bar{d}(B)=\bar{d}_{m}(B)$ ) подгруппу групшы $\mathrm{Br}_{2 m}$, порожденную элементами, принадлежашими группам $B, \operatorname{sh}_{m}(B)$, и элементами $c_{m, 1}, \ldots, c_{m, m}$ (соответственно, порожденную элементами, принадлежащими групе $B$, и элементами $c_{m, 1}, \ldots, c_{m, m}$ ), и назовем ее удвоением (соответственно, приведенным удвоением) группы $B$.

Удвоение (соответственно, приведенное удвоение) группы $B$ может быть итерировано, если положить

$$
d^{n}(B)=d_{2^{n-1} m}\left(d^{n-1}(B)\right) \subset \operatorname{Br}_{2^{n} m}
$$

УТВЕРЖДЕНИЕ 1.13. Для любого $n \in \mathbb{N}$ множества соотношений $R_{W\left(B, \mathbb{F}_{m}\right)}$, $R_{d^{n}\left(W\left(B, \mathbb{F}_{m}\right)\right)}, R_{\bar{d}^{n}\left(W\left(B, \mathbb{F}_{m}\right)\right)}, R_{W\left(d^{n}(B), \mathbb{F}_{2^{n}}\right)} u R_{W\left(\bar{d}^{n}(B), \mathbb{F}_{2}{ }_{m}\right)}$ являяются әквивалентными. 
ДоКАЗАТЕЛЬСТвО следует из утверждений 1.4 и 1.12.

Скажем, что $C$-группа $G$ принадлежит подклассу $\mathscr{C}_{\mathrm{Br}}$, если она $C$-изоморфна группе, заданной копредставлением

$$
G=\left\langle x_{1}, \ldots, x_{m} \mid R_{W\left(B, \mathbb{F}_{m}\right)}\right\rangle
$$

для некоторого $m$ и некоторой конечно порожденной подгрупшы $B$ групшы кос $\mathrm{Br}_{m}$.

ТЕорема 1.14. Для произвольной $C$-группь $G$ найдутся положительное челое число $M=M_{G} \in \mathbb{Z}$ и подгруппа $B_{G}$ группь $\mathrm{Br}_{M}$, порожденная конечным множеством $\left\{b_{1}, \ldots, b_{n_{G}}\right\}$ әлементов, сопряженных в $\mathrm{Br}_{M}$ стандартной образующей $a_{1}$, и такая, ито группа $G$ является $C$-изоморфной группе $G\left(W\left(B, \mathbb{F}_{M}\right)\right)$.

ДоКАЗАТЕЛЬСТво следует из утверждений 1.4, 1.7, 1.8, 1.11 и 1.12.

СлЕДСТВИЕ 1.15. Имеем $\mathscr{C}_{\mathrm{Br}}=\mathscr{C}$.

Напомним также следуюшую теорему.

Teорема 1.16 [1]. (i) C-группа $G$ принадлежит $\mathscr{L}$ тогда и только тогда, когда найдутся иелое число $m$ и әлемент $b \in \mathrm{Br}_{m}$ такой, что группа $G$ С-изоморфна группе

$$
G(W(\langle b\rangle, \bar{x}))=\left\langle x_{1}, \ldots, x_{m} \mid x_{i}=b\left(x_{i}\right), i=1, \ldots, m\right\rangle .
$$

(ii) $C$-группа $G$ с копредставлением (13) принадлежит классу $\mathscr{K}$ тогда и только тогда, когда подстановка $\sigma_{b} \in \mathscr{S}_{m}$ состоит из одного иикла длины $m$, m.е. $\sigma_{b}$ действует транзитивно на множестве $\{1, \ldots, m\}$.

\section{§2. Разложение на множители полного поворота}

Хорошо известно, что элемент (назьваемый полным поворотом)

$$
\Delta_{m}^{2}=\left(a_{1} \ldots a_{m-1}\right)^{m}
$$

порождает центр группы кос $\mathrm{Br}_{m}$. Имеем

$$
\Delta_{m}^{2}\left(x_{i}\right)=\left(x_{1} \ldots x_{m}\right) x_{i}\left(x_{1} \ldots x_{m}\right)^{-1} .
$$

В частности, полный поворот $\Delta_{m}^{2}$ оставляет на месте элемент $l_{m}=x_{1} \ldots x_{m}$.

Рассмотрим группу кос $\mathrm{Br}_{2 m}$. Положим

$$
\begin{aligned}
\bar{r}_{m} & =\bar{c}_{m, m} \bar{c}_{m, m-1} \ldots \bar{c}_{m, 1}, \\
r_{m} & =c_{m, m} c_{m, m-1} \ldots c_{m, 1},
\end{aligned}
$$

где входящие в формулы $\bar{c}_{m, i}$ и $c_{m, i}$ были определены соотношениями (10) и (11).

Лемма 2.1. В группе кос $\mathrm{Br}_{2 m}$ имеет место равенство

$$
\Delta_{2 m}=\Delta_{m, 0}^{2} \Delta_{m, m}^{2} \bar{r}_{m}
$$


ДокАЗАТЕЛЬСТво. Отождествим группу $\mathrm{Br}_{2(m-1)}$ с подгруппой $B_{2(m-1), 1}$ группы $\mathrm{Br}_{2 m}$. Используя индукцию по $m$, имеем

$$
\bar{r}_{m}=\bar{c}_{m, m} \bar{c}_{m, m-1} \ldots \bar{c}_{m, 2} \bar{c}_{m, 1}=\Delta_{m-1, m}^{-2} \Delta_{m-1,1}^{-2} \Delta_{2(m-1), 1} \bar{c}_{m, 1} .
$$

Следовательно, чтобы доказать лемму, достаточно показать, что действия на $\mathbb{F}_{2 m}$ элементов $\Delta_{2 m}$ и

$$
b=\Delta_{m, 0}^{2} \Delta_{m, m}^{2} \Delta_{m-1, m}^{-2} \Delta_{m-1,1}^{-2} \Delta_{2(m-1), 1} \bar{c}_{m, 1}
$$

совпадают между собой.

Зафиксируем базис $\left\{x_{1}, \ldots, x_{2 m}\right\}$ группы $\mathbb{F}_{2 m}$, изображенный на рис. 1 . В этом случае действие элемента $\bar{c}_{m, 1}$ на $\mathbb{F}_{2 m}$ индуцируется полуповоротом, определяемым путем $\gamma_{m, 1}$, которьй изображен на рис. 3.

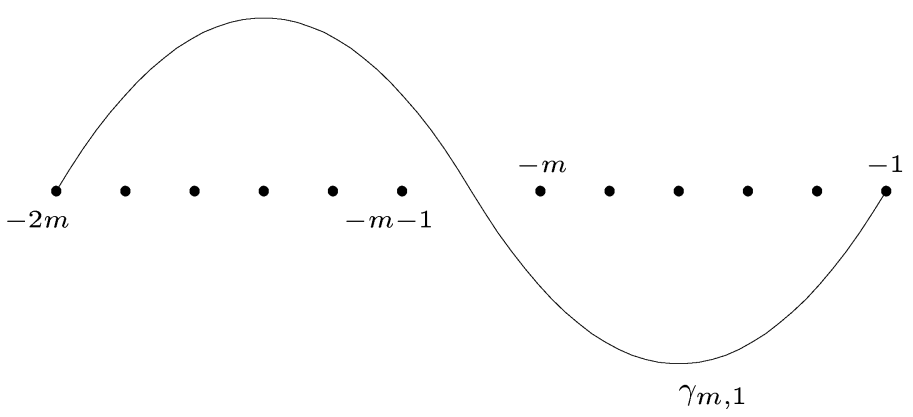

Рис. 3

Следовательно, элемент $y_{1}=\bar{c}_{m, 1}\left(x_{1}\right)$ представляется петлей, изображенной на рис. 4.

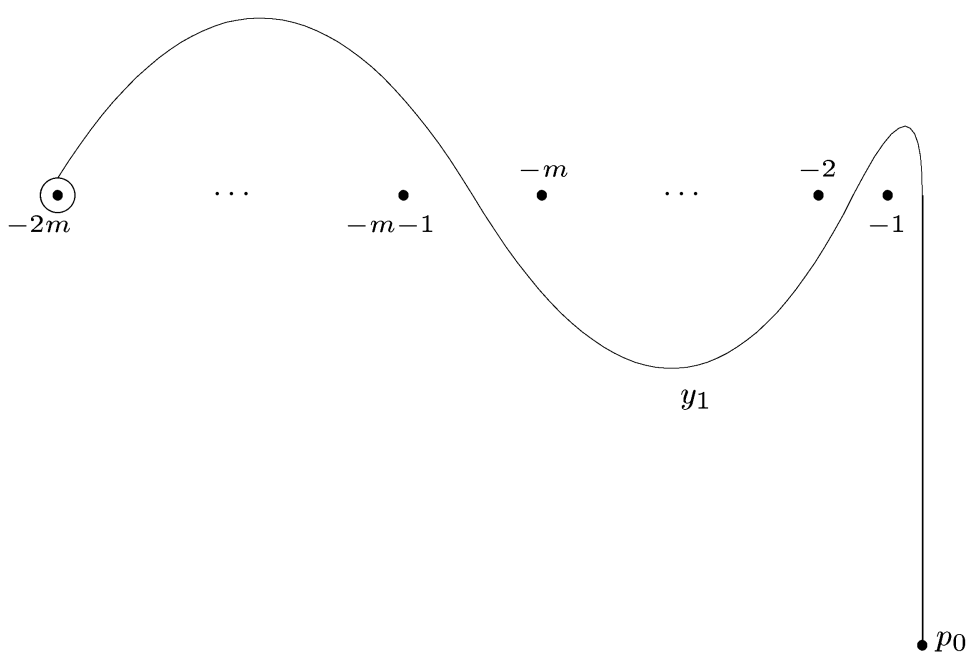

Рис. 4 
Элемент $u_{1}=\Delta_{2(m-1), 1}\left(y_{1}\right)$ представляется петлей, изображенной на рис. 5 .

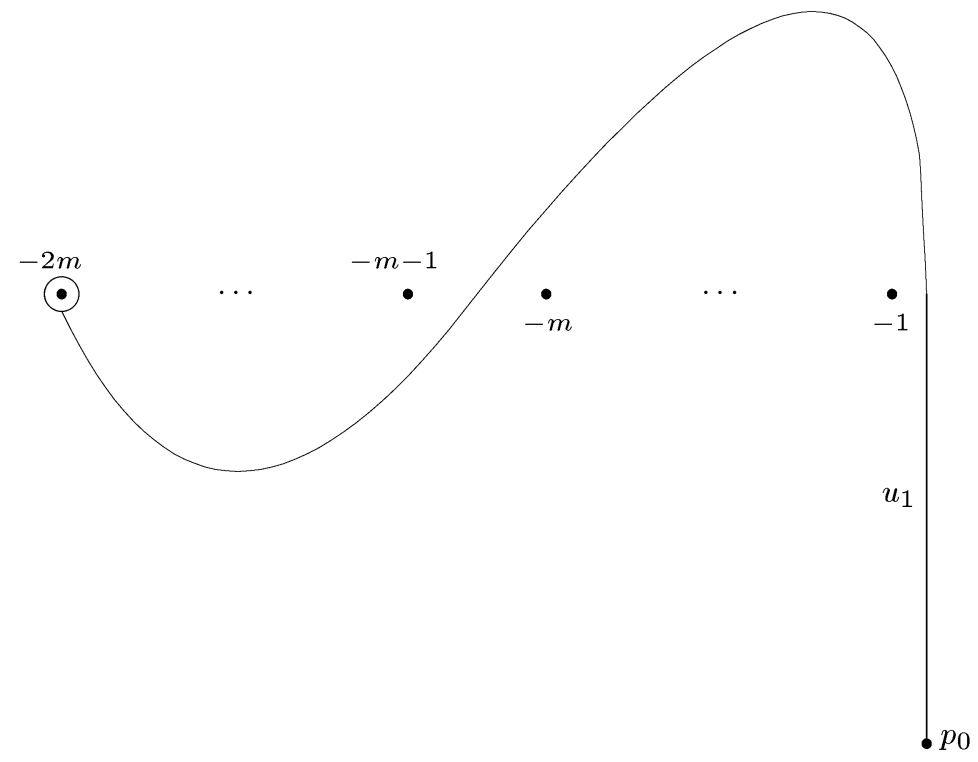

Рис. 5

Легко видеть, что $\Delta_{m-1, m}^{-2} \Delta_{m-1,1}^{-2}\left(u_{1}\right)=u_{1}$, а элемент $v_{1}=\Delta_{m, 0}^{2} \Delta_{m, m}^{2}\left(u_{1}\right)=$ $b\left(x_{1}\right)$ представляется петлей, изображенной на рис. 6.

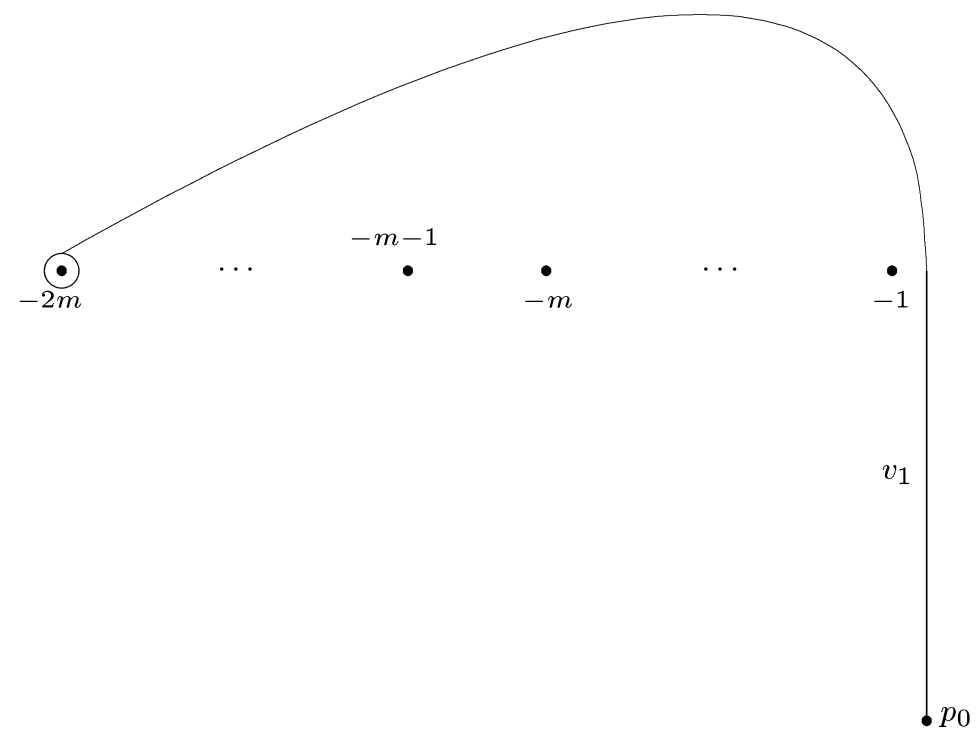

Рис. 6

Следовательно, согласно равенству (7) имеем

$$
b\left(x_{1}\right)=\Delta_{2 m}\left(x_{1}\right) .
$$


Элемент $y_{i}=\bar{c}_{m, 1}\left(x_{i}\right), i=2, \ldots, m$, представляется петлей, изображенной на рис. 7.

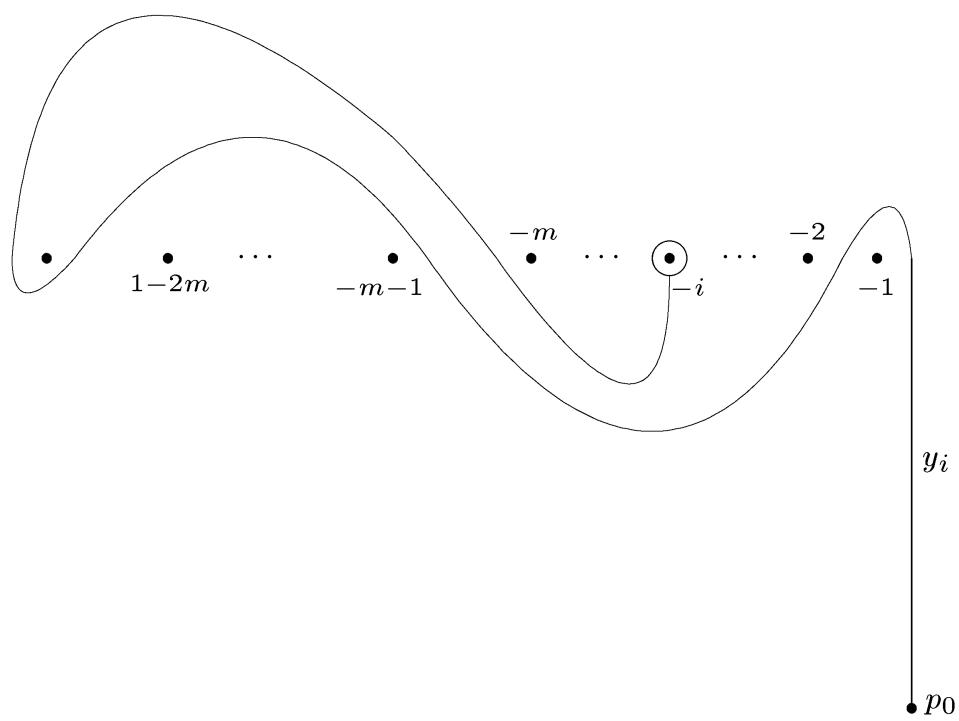

Рис. 7

Элемент $u_{i}=\Delta_{2(m-1), 1}\left(y_{i}\right), i=2, \ldots, m$, представляется петлей, изображенной на рис. 8.

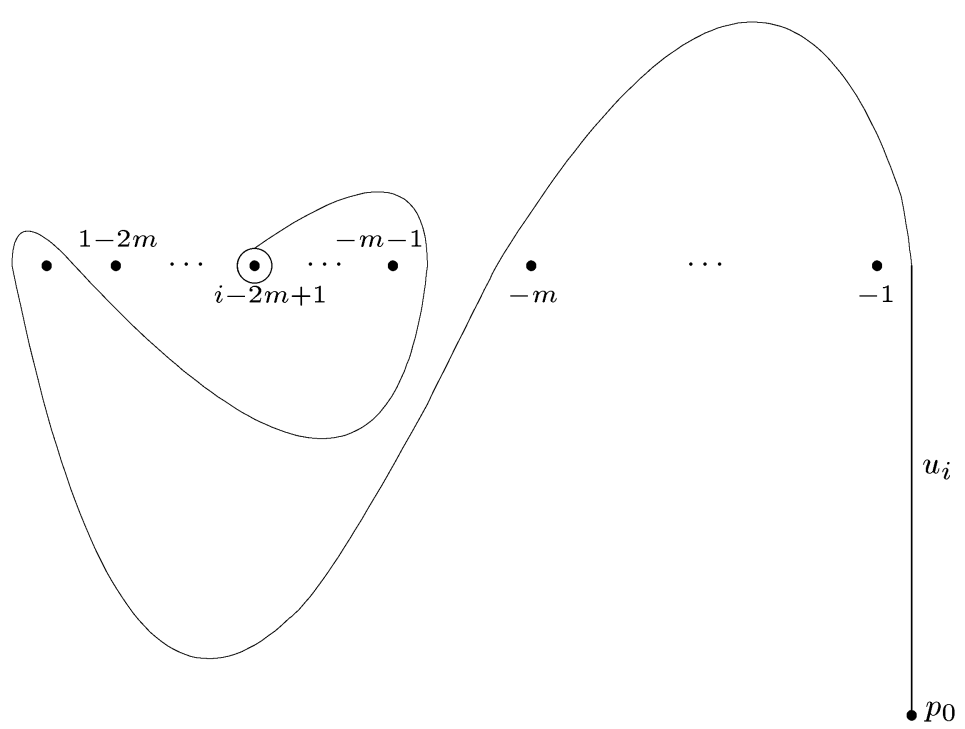

Рис. 8 
Элемент $v_{i}=\Delta_{m-1, m}^{-2} \Delta_{m-1,1}^{-2}\left(u_{i}\right), i=2, \ldots, m$, представляется петлей, изображенной на рис. 9.

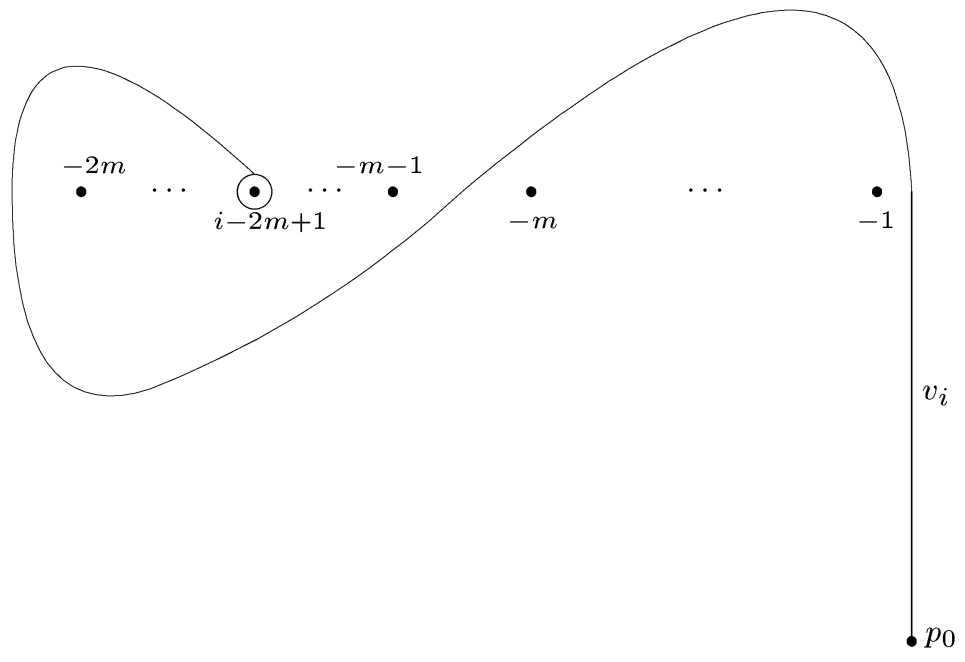

Рис. 9

Элемент $w_{i}=\Delta_{m, 0}^{2} \Delta_{m, m}^{2}\left(v_{i}\right)=b\left(x_{i}\right), i=2, \ldots, m$, представляется петлей, изображенной на рис. 10.

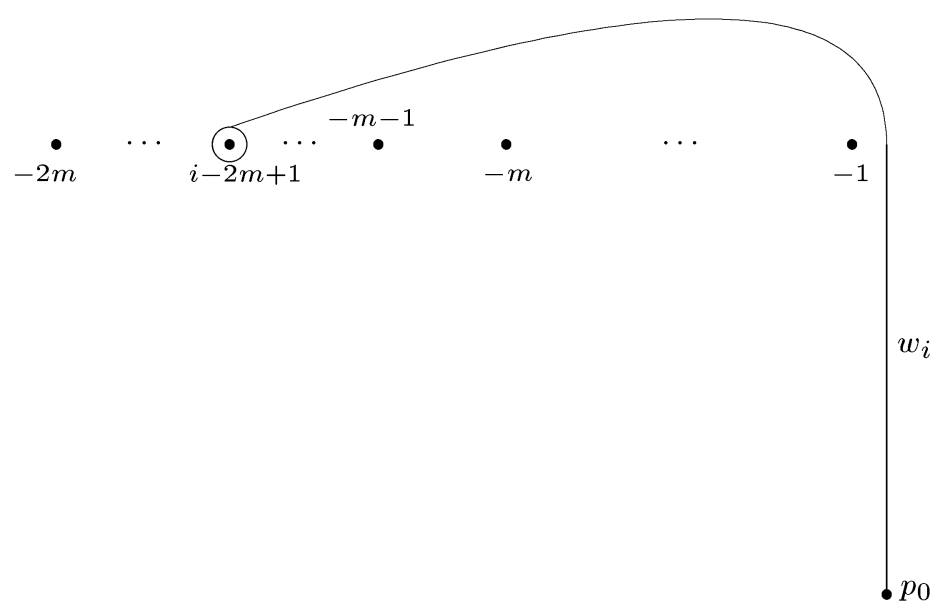

Рис. 10

Следовательно,

$$
b\left(x_{i}\right)=\Delta_{2 m}\left(x_{i}\right), \quad i=2, \ldots, m .
$$


Легко видеть, что $\bar{c}_{m, 1}\left(x_{2 m-i}\right)=x_{2 m-i}$ для $i=1, \ldots, m-1$. Элемент $y_{2 m-i}=$ $\Delta_{2(m-1), 1}\left(x_{2 m-i}\right)$ представляется петлей, изображенной на рис. 11.

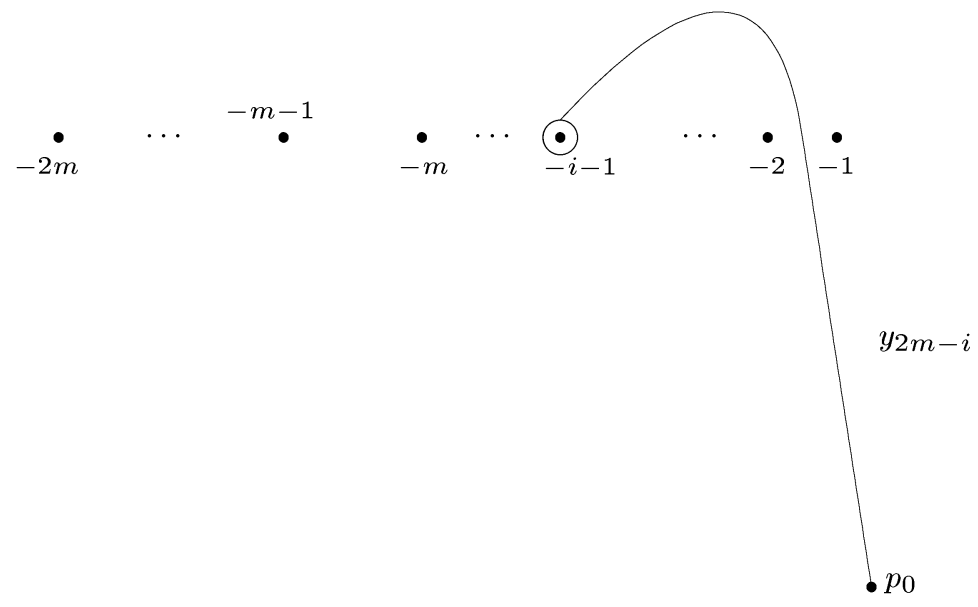

Рис. 11

Элемент $u_{2 m-i}=\Delta_{m-1, m}^{-2} \Delta_{m-1,1}^{-2}\left(y_{2 m-i}\right)$ представляется петлей, изображенной на рис. 12.

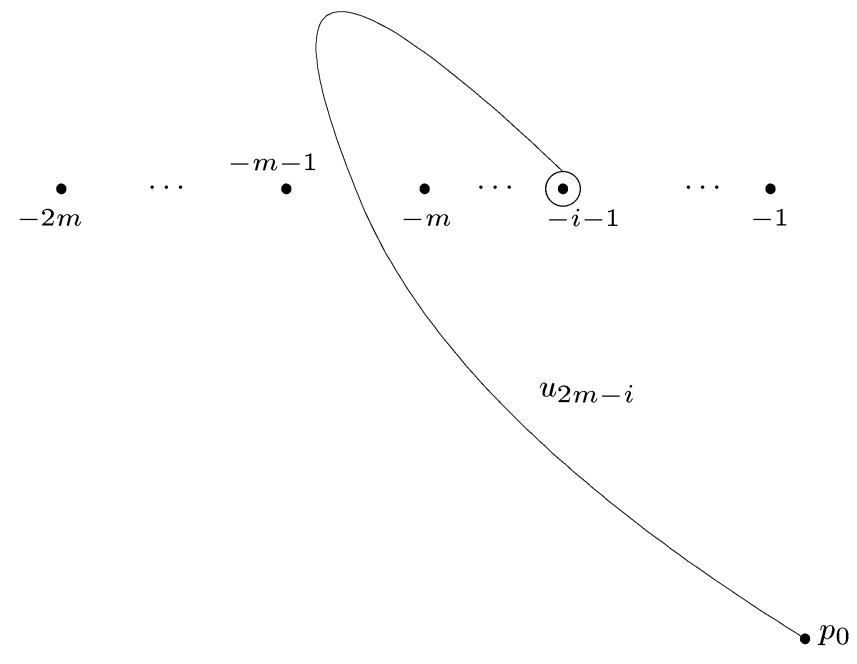

Рис. 12

Элемент $v_{2 m-i}=\Delta_{m, 0}^{2} \Delta_{m, m}^{2}\left(u_{2 m-i}\right)=b\left(x_{2 m-i}\right)$ представляется петлей, изображенной на рис. 13. 


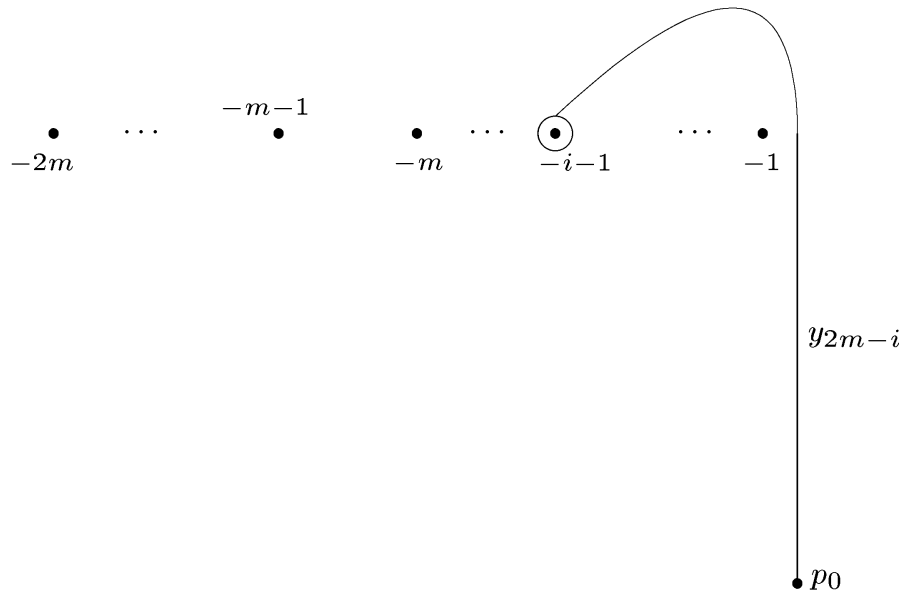

Рис. 13

Следовательно, согласно равенству (7) имеем

$$
b\left(x_{2 m-i}\right)=\Delta_{2 m}\left(x_{2 m-i}\right), \quad i=1, \ldots, m-1 .
$$

Элемент $y_{2 m}=\bar{c}_{m, 1}\left(x_{2 m}\right)$ представляется петлей, изображенной на рис. 14.

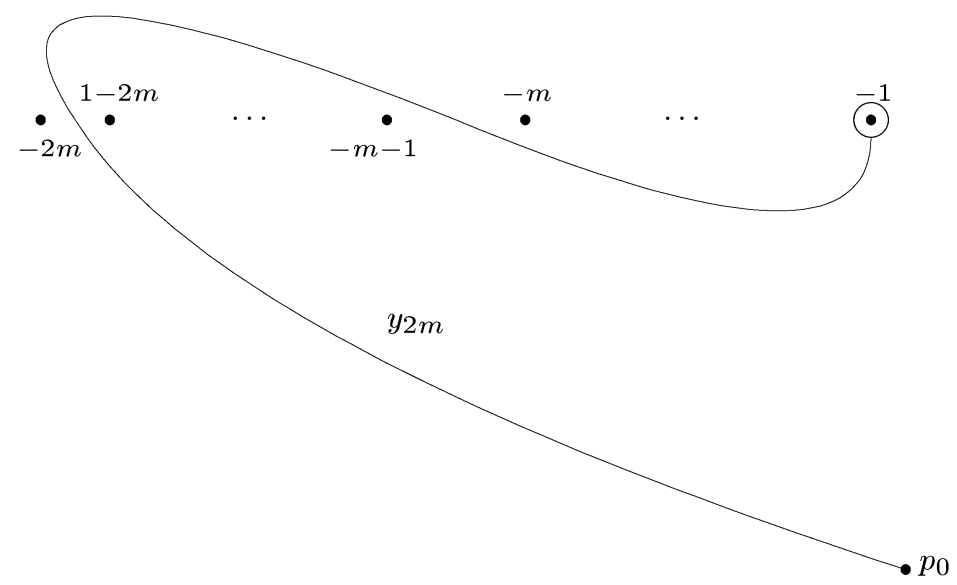

Рис. 14

Элемент $u_{2 m}=\Delta_{2(m-1), 1}\left(y_{2 m}\right)$ представляется петлей, изображенной на рис. 15.

Легко видеть, что $\Delta_{m-1, m}^{-2} \Delta_{m-1,1}^{-2}\left(u_{2 m}\right)=u_{2 m}$, и элемент

$$
b\left(x_{2 m}\right)=\Delta_{m, 0}^{2} \Delta_{m, m}^{2}\left(u_{2 m}\right)=x_{1} .
$$




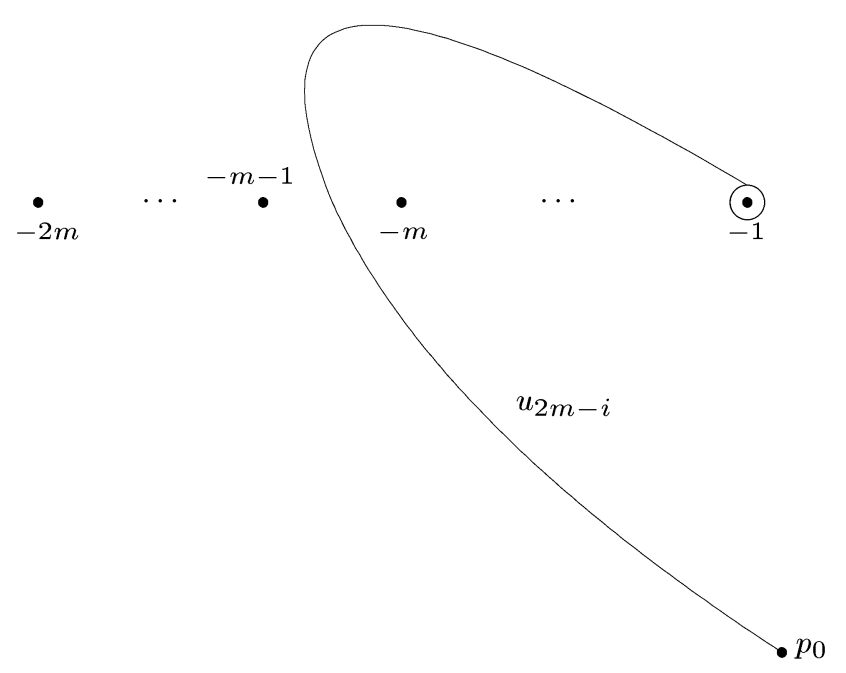

Рис. 15

Следовательно,

$$
b\left(x_{2 m}\right)=\Delta_{2 m}\left(x_{2 m}\right)
$$

и лемма следует из равенств (16)-(19).

Теорема 2.2. Имеет место следующая формула разложения на множители полного поворота при удвоении числа нитей:

$$
\Delta_{2 m}^{2}=\Delta_{m, 0}^{4} \Delta_{m, m}^{4} r_{m}^{2}
$$

ДоКАЗАТЕЛЬСТВо. Согласно лемме 2.1

$$
\Delta_{2 m}=\Delta_{m, 0}^{2} \Delta_{m, m}^{2} \bar{r}_{m}
$$

Легко видеть, что $\left[\Delta_{m, 0}, \Delta_{m, m}\right]=\mathbf{1}$ и $\left[\Delta_{2 m}, \Delta_{m, 0}^{2} \Delta_{m, m}^{2}\right]=\mathbf{1}$. Следовательно, $\left[\bar{r}_{m, m}, \Delta_{m, 0}^{2} \Delta_{m, m}^{2}\right]=\mathbf{1}$ и

$$
\Delta_{2 m}^{2}=\Delta_{m, 0}^{4} \Delta_{m, m}^{4} \bar{r}_{m}^{2}
$$

Таким образом,

$$
\Delta_{2 m}^{2}=\Delta_{m, m}^{-1} \Delta_{2 m}^{2} \Delta_{m, m}=\Delta_{m, 0}^{4} \Delta_{m, m}^{4} r_{m}^{2}
$$

\section{§ 3. Полугруппы разложений на множители над группами кос}

Пусть $\left\{g_{i}\right\}_{i \in I}$ - некоторое множество элементов групшы $\mathrm{Br}_{m}$. Для каждого $i \in I$ обозначим через $O_{g_{i}} \subset \mathrm{Br}_{m}$ множество всех элементов в $\mathrm{Br}_{m}$, сопряженных элементу $g_{i}$ (орбиту элемента $g_{i}$ при действии группы $\mathrm{Br}_{m}$ внутренними автоморфизмами). Объединение $U=\bigcup_{i \in I} O_{g_{i}} \subset \mathrm{Br}_{m}$ называется полным множсеством сопряжсенных элементам из $\left\{g_{i}\right\}_{i \in I}$, а пара $\left(\mathrm{Br}_{m}, U\right)$ - оснащенной 
әруппой кос. В работе [7] каждой оснащенной групе $\left(\mathrm{Br}_{m}, U\right)$ была сопоставлена полугруппа $S\left(\mathrm{Br}_{m}, U\right)$, названная полугруппой разложений на множители над $\mathrm{Br}_{m}$. Напомним, что по определению, данному в [7], полугруппа $S\left(\mathrm{Br}_{m}, U\right)$ порождена алфавитом $U$, удовлетворяюшим следующим соотношениям:

$$
\begin{aligned}
& u_{1} \cdot u_{2}=u_{2} \cdot\left(u_{2}^{-1} u_{1} u_{2}\right), \text { если } u_{2} \neq \mathbf{1} \text { и } u_{1} \cdot \mathbf{1}=u_{1}, \\
& u_{1} \cdot u_{2}=\left(u_{1} u_{2} u_{1}^{-1}\right) \cdot u_{1}, \text { если } u_{1} \neq \mathbf{1} \text { и } \mathbf{1} \cdot u_{2}=u_{2},
\end{aligned}
$$

для всех $u_{1}, u_{2} \in U$.

Существуют два естественных гомоморфизма: гомоморфизм произведения $\alpha=$ $\alpha_{U}: S\left(\mathrm{Br}_{m}, U\right) \rightarrow \mathrm{Br}_{m}$, заданный условием $\alpha(u)=u$ для всех $u \in U$, и гомоморфизм (действие сопряжсением) $\lambda: \operatorname{Br}_{m} \rightarrow \operatorname{Aut}\left(S\left(\mathrm{Br}_{m}, U\right)\right)$, заданньй равенством $\lambda(g)(u)=g u g^{-1} \in U$ для всех $g \in \mathrm{Br}_{m}$. Действие $\lambda(g)$ на $S\left(\mathrm{Br}_{m}, U\right)$ называется одновременным сопряжением на элемент $g$. Положим $\rho(g)=\lambda\left(g^{-1}\right)$, $\lambda_{S}=\lambda \circ \alpha_{U}$ и $\rho_{S}=\rho \circ \alpha_{U}$. Орбита элемента $s \in S\left(\mathrm{Br}_{m}, U\right)$ при действии сопряжением групшы $\mathrm{Br}_{m}$ на $S\left(\mathrm{Br}_{m}, U\right)$ называется типом элемента $s$.

Отметим, что $S:\left(\mathrm{Br}_{m}, U\right) \mapsto\left(S\left(\mathrm{Br}_{m}, U\right), \alpha_{U}, \lambda\right)$ является функтором из категории оснащенных групп кос в категорию полугрупп над группами кос. В частности, если $U \subset V$ - два полных множества сопряженных элементов в $\mathrm{Br}_{m}$, то тождественное отображение id: $\mathrm{Br}_{m} \rightarrow \mathrm{Br}_{m}$ определяет вложение $\mathrm{id}_{U, V}: S\left(\mathrm{Br}_{m}, U\right) \rightarrow$ $S\left(\mathrm{Br}_{m}, V\right)$. Таким образом, для каждой группы $\mathrm{Br}_{m}$ полугруппа $S_{\mathrm{Br}_{m}}=S\left(\mathrm{Br}_{m}\right.$, $\mathrm{Br}_{m}$ ) является универсальной полугруппой над $\mathrm{Br}_{m}$, т.е. каждая полугруппа разложений на множители $S_{U}=S\left(\mathrm{Br}_{m}, U\right)$ над $\mathrm{Br}_{m}$ канонически вкладывается в $S_{\mathrm{Br}_{m}}$ с помошью отображения $\mathrm{id}{ }_{U, \mathrm{Br}_{m}}$.

Поскольку $\alpha_{U}=\alpha_{\mathrm{Br}_{m}} \circ \mathrm{id}_{U, \mathrm{Br}_{m}}$, гомоморфизмы произведения $\alpha_{U}$ и $\alpha_{\mathrm{Br}_{m}}$ могут быть отождествлены, так что они оба будут обозначаться просто $\alpha$.

Для любых $s_{1}, s_{2} \in S\left(\mathrm{Br}_{m}, U\right)$ имеем

$$
s_{1} \cdot s_{2}=s_{2} \cdot \rho_{S}\left(s_{2}\right)\left(s_{1}\right)=\lambda_{S}\left(s_{1}\right)\left(s_{2}\right) \cdot s_{1} .
$$

Скажем, что элемент $b \in \mathrm{Br}_{m}$ имеет индекс сплетения $l(b)=k$, если $k-$ это такое наименьшее число, что элемент $b$ сопряжен в $B_{m}$ некоторому элементу $\bar{b} \in B_{k, 0}, b=g \bar{b} g^{-1}$. Элемент $\bar{b}$ называется стандартной формой элемента $b$.

Для каждого $s \in S_{U}$ обозначим через $B_{s}$ подгруппу группы $\mathrm{Br}_{m}$, порожденную образами $\alpha\left(u_{1}\right), \ldots, \alpha\left(u_{n}\right)$ множителей $u_{1}, \ldots, u_{n}$ разложения $s=u_{1} \cdot \ldots \cdot u_{n}$. Легко видеть, что подгруппа $B_{s}$ группы $\mathrm{Br}_{m}$ не зависит от представления элемента $s \in S_{U}$ в виде слова, состояшего из букв $u_{i} \in U$.

Свяжем с элементом $s \in S_{B_{m}}$ еще одну группу

$$
\begin{aligned}
G(s) & =G\left(W_{s}\right)=G\left(W\left(B_{s}, \mathbb{F}_{m}\right)\right) \\
& \simeq\left\langle x_{1}, \ldots, x_{m} \mid x_{i}^{-1} b\left(x_{i}\right)=\mathbf{1}, i=1, \ldots, m, b \in B_{s}\right\rangle .
\end{aligned}
$$

УТВЕРЖДЕНИЕ 3.1. Для $s_{1}, s_{2} \in S_{\mathrm{Br}_{m}}$ имеем:

(i) существует канонический $C$-эпиморфизм

$$
\psi_{s_{1}}: G\left(s_{2}\right) \rightarrow G\left(s_{1} \cdot s_{2}\right) \simeq G\left(s_{2}\right) / N_{G\left(s_{2}\right)}\left(\varphi_{W_{s_{2}}}\left(W_{s_{1}}\right)\right)
$$

(ii) если $s_{2}=\lambda(b)\left(s_{1}\right)$, то существует $C$-изоморфизм групп $\gamma_{b}: G\left(s_{1}\right) \rightarrow$ $G\left(s_{2}\right) ;$ в частности, если $s_{1}=s_{2}$, то $G\left(s_{1}\right)=G\left(s_{2}\right)$ и $\gamma_{\mathbf{1}}=\mathrm{Id}$. 
ДокАЗАТЕЛЬСТво проводится прямой проверкой.

Пусть множитель $u_{i}$ элемента $s=u_{1} \cdot \ldots \cdot u_{n}$ имеет индекс сплетения $l\left(u_{i}\right)=k_{i}$ и стандартную форму $\bar{u}_{i} \in \mathrm{Br}_{k, 0}, u_{i}=g_{i} \bar{u}_{i} g_{i}^{-1}$. Обозначим через $G_{u_{i}, \text { loc подгруппу }}$ в $G(s)$, порожденную элементами $\varphi_{W_{s}}\left(g_{i}\left(x_{j}\right)\right), j=1, \ldots, k_{i}$. Из $(21)$ следует, что

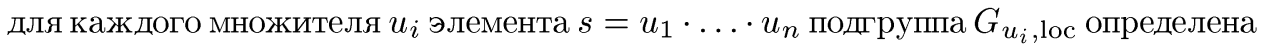
однозначно с точностью до сопряжения в $G(s)$.

Вложение $\mathrm{Br}_{m}=B_{m, 0} \subset \mathrm{Br}_{2 m}$ индуцирует вложение $S_{\mathrm{Br}_{m}} \subset S_{\mathrm{Br}_{2 m}}$. Рассмотрим гомоморфизм $\mathrm{sh}=\mathrm{sh}_{m}: \operatorname{Br}_{m} \rightarrow \operatorname{Br}_{2 m}$, заданный равенствами $\operatorname{sh}\left(a_{i}\right)=a_{m+i}$ для $i=1, \ldots, m-1$. Он индуцирует вложение $\mathrm{sh}: S_{\mathrm{Br}_{m}} \rightarrow S_{\mathrm{Br}_{2 m}}$. Положим также

$$
\tilde{r}_{m}=c_{m, m} \cdot \ldots \cdot c_{m, 1} \in S_{\mathrm{Br}_{2 m}}
$$

где $c_{m, i}$ определены формулой (11). Применяя теорему 2.2, получаем следующую теорему.

ТЕОРЕМА 3.2 (формула разложения на множители полного поворота для удвоенного числа нитей). Для четырех әлементов $s_{1}, \ldots, s_{4} \in S_{\mathrm{Br}_{m}}$ таких, что $\alpha\left(s_{i}\right)=\Delta_{m}^{2}$, әлемент

$$
\bar{s}=d\left(s_{1}, s_{2}, s_{3}, s_{4}\right)=s_{1} \cdot s_{2} \cdot \operatorname{sh}\left(s_{3}\right) \cdot \operatorname{sh}\left(s_{4}\right) \cdot \tilde{r}_{m} \cdot \tilde{r}_{m}
$$

является разложением әлемента $\Delta_{2 m}^{2}$ на множители в полугруппе $S_{\mathrm{Br}_{2 m}}$, m. e. $\alpha(\bar{s})=\Delta_{2 m}^{2}$.

Если $s_{1}=s_{2}=s_{3}=s_{4}=s$, удвоение $d(s, s, s, s)$ будем обозначать просто через

$$
d(s)=d(s, s, s, s)=s \cdot s \cdot \operatorname{sh}(s) \cdot \operatorname{sh}(s) \cdot \tilde{r}_{m} \cdot \tilde{r}_{m}
$$

и положим

$$
d^{n+1}(s)=d\left(d^{n}(s)\right) .
$$

УТВЕРЖДЕНИЕ 3.3. Для $s \in S_{\mathrm{Br}_{m}}$ такого, что $\alpha(s)=\Delta_{m}^{2}$, группы $G(s) u$ $G(d(s))$ являются $C$-изоморфньми.

ДокАЗАТЕЛЬСТво следует из утверждения 1.13.

Лемма 3.4. Пусть элемент $s \in S_{\mathrm{Br}_{M}}$ такой, что $\alpha(s)=\Delta_{M}^{2}$. Рассмотрим әлемент $g \in \mathrm{Br}_{M}$, для которого $g\left(x_{2}\right)=x_{i}$ при некотором $i \leqslant M$. Положим $y=g\left(x_{1}\right) \in \mathbb{F}_{M} u b=g a_{1} g^{-1}$. Тогда:

(i) $C$-әруппа $\bar{G}=G(s) / N_{G(s)}\left(\varphi_{W_{s}}\left(x_{i}^{-1} y\right)\right)$ канонически $C$-изоморфна групne $G(\bar{s})$, əде

$$
\bar{s}=d\left(\lambda_{S}(b)(d(s)), d(s), d(s), d(s)\right) ;
$$

(ii) $C$-әруппа $\widetilde{G}=G(s) / N_{G(s)}\left(\varphi_{W_{s}}\left(\left[x_{i}, y\right]\right)\right)$ канонически $C$-изоморфна групne $G(\tilde{s})$, әде

$$
\tilde{s}=d\left(\lambda_{S}\left(b^{2}\right)(d(s)), d(s), d(s), d(s)\right) .
$$

Если $M=m k$ и множество соотношений $R_{W_{s}}$ влечет соотношения $R_{\bmod m} \boldsymbol{\theta} \mathbb{F}_{M}$, то множсество соотношений $R_{W_{\bar{s}}}$ (соответственно, $\left.R_{W_{\tilde{s}}}\right)$ влечет соотношения $R_{\bmod m}$ в $\mathbb{F}_{4 M}$. 
ДокАЗАТЕЛЬСТво. Согласно утверждению $3.3 C$-группа $G\left(d^{2}(s)\right)$ канонически $C$-изоморфна группе $G(s)$. Применяя утверждение 1.10 , получаем, что множество соотношений $R_{W\left(\left\langle b^{k}\right\rangle, \mathbb{F}_{M}\right)}$ эквивалентно одному соотношению: или $\left\{x_{i}^{-1} y=\mathbf{1}\right\}$, если $k=1$, или $\left\{\left[x_{i}, y\right]=\mathbf{1}\right\}$, если $k=2$. Согласно утверждению $3.1 C$-группа $\bar{G}$ канонически изоморфна группе $G\left(b \cdot d^{2}(s)\right)$, а $C$-группа $\widetilde{G}$ канонически изоморфна группе $G\left(b^{2} \cdot d^{2}(s)\right)$.

Положим

$$
s^{\prime}=d(s) \cdot \operatorname{sh}_{2 M}(d(s)) \cdot \operatorname{sh}_{2 M}(d(s)) \cdot \tilde{r}_{2 M} \cdot \tilde{r}_{2 M} .
$$

Элемент

$$
\begin{aligned}
b^{k} \cdot d^{2}(s) & =b^{k} \cdot d(s) \cdot s^{\prime}=\lambda_{S}\left(b^{k}\right)(d(s)) \cdot b^{k} \cdot s^{\prime} \\
& =\lambda_{S}\left(\lambda_{S}\left(b^{k}\right)(d(s))\right)\left(b^{k}\right) \cdot \lambda_{S}\left(b^{k}\right)(d(s)) \cdot s^{\prime} \\
& =b^{k} \cdot \lambda_{S}\left(b^{k}\right)(d(s)) \cdot s^{\prime}= \begin{cases}b \cdot \bar{s}, & \text { если } k=1, \\
b^{2} \cdot \tilde{s}, & \text { если } k=2,\end{cases}
\end{aligned}
$$

так как

$$
\alpha(d(s))=\alpha\left(\lambda_{S}\left(b^{k}\right)(d(s))\right)=\Delta_{2 M}^{2}
$$

и элементы $b^{k}$ и $\Delta_{2 M}^{2}$ коммутируют в $\mathrm{Br}_{2 M}$. Следовательно, согласно утверждению 3.1 существуют канонические эпиморфизмы $\psi_{b}: G(\bar{s}) \rightarrow \bar{G}$ и $\psi_{b^{2}}: G(\tilde{s}) \rightarrow \widetilde{G}$. $\mathrm{C}$ другой стороны, множители элемента $\bar{s}$, входяшие в произведение

$$
d(s) \cdot \operatorname{sh}_{2 M}(d(s)) \cdot \operatorname{sh}_{2 M}(d(s)) \cdot \tilde{r}_{2 M} \cdot \tilde{r}_{2 M},
$$

определяют то же множество соотношений, что и элемент $d^{2}(s)$. В частности, эти соотношения влекут соотношения $\left\{x_{M+i}=x_{i}\right\} \in R_{\bmod M}$ в $\mathbb{F}_{4 M}$, и, более того, если множество соотношений $R_{W_{s}}$ влечет соотношения $R_{\bmod m}$ в $\mathbb{F}_{M}$, то множество соотношений $R_{W_{\tilde{s}}}$ (соответственно, $R_{W_{\tilde{s}}}$ влечет соотношения $R_{\bmod } m$ в $\mathbb{F}_{4 M}$. Множитель $\lambda_{S}\left(b^{k}\right)\left(c_{M, i}\right)=b^{k} c_{M, i} b^{-k}$ элемента $\lambda_{S}\left(b^{k}\right)(d(s))$ определяет соотношение $x_{M+i}=b c_{M, i} b^{-1}\left(x_{M+i}\right)$ в $G(\bar{s})$, если $k=1$, и соотношение $x_{M+i}=$ $b^{2} c_{M, i} b^{-2}\left(x_{M+i}\right)$ в $G(\tilde{s})$, если $k=2$. Однако

$$
b^{k} c_{M, i} b^{-k}\left(x_{M+i}\right)=b^{k} c_{M, i}\left(x_{M+i}\right)=b^{k}\left(x_{i}\right) .
$$

Для $k=1,2$ имеем $b\left(x_{i}\right)=y$ и $b^{2}\left(x_{i}\right)=y x_{i} y^{-1}$. Соотношения $\left\{x_{M+i}=y\right\}$ (соответственно, $\left.\left\{x_{M+i}=y x_{i} y^{-1}\right\}\right)$ и $\left\{x_{M+i}=x_{i}\right\}$ влекут соотношение $x_{i}=y$ в $G(\bar{s})$ (соответственно, $\left\{\left[x_{i}, y\right]=\mathbf{1}\right\}$ в $\left.G(\tilde{s})\right)$. Следовательно, канонические эпиморфизмы $\psi_{b}: G(\bar{s}) \rightarrow \bar{G}$ и $\psi_{b^{2}}: G(\tilde{s}) \rightarrow \widetilde{G}$ являются $C$-изоморфизмами.

Рассмотрим подклассы $\mathscr{C}_{\Delta^{2}, m}, m \in \mathbb{N}$, класса групп $\mathscr{C}$, состоящие из $C$-групп, которые являются $C$-изоморфными $C$-группам, обладающим $C$-копредставлениями вида

$$
G=\left\langle x_{1}, \ldots, x_{m} \mid x_{i}^{-1} w_{i, j, k}(\bar{x})^{-1} x_{j} w_{i, j, k}(\bar{x})=\mathbf{1}, w_{i, j, k}(\bar{x}) \in W\right\rangle
$$

для некоторого конечного множества $W \subset \mathbb{F}_{m}$ такого, что слова $\left[x_{i}^{-1}, x_{1} \ldots x_{m}\right]$ принадлежат множеству $W$ для всех $i=1, \ldots, m$. Положим

$$
\mathscr{C}_{\Delta^{2}}=\bigcup_{m \in \mathbb{N}} \mathscr{C}_{\Delta^{2}, m} \text {. }
$$


УТВЕРЖДЕНИЕ 3.5. Пусть $s \in S_{\mathrm{Br}_{m}}-$ такой әлемент, что $\alpha(s)=\Delta_{m}^{2}$; тогда $C$-группа $G(s) \in \mathscr{C}_{\Delta^{2}, m}$. В частности, для $s=\Delta_{m}^{2}$ группа $G\left(\Delta_{m}^{2}\right)$ имеет $C$-копредставление

$$
G\left(\Delta_{m}^{2}\right)=\left\langle x_{1}, \ldots, x_{m} \mid\left[x_{i}^{-1}, x_{1} \ldots x_{m}\right]=\mathbf{1}, i=1, \ldots, m\right\rangle .
$$

ДокаЗАТЕЛЬСтво. Имеем $\Delta_{m}^{2}\left(x_{i}\right)=\left(x_{1} \ldots x_{m}\right) x_{i}\left(x_{1} \ldots x_{m}\right)^{-1}$. Следовательно, соотношения $\left[x_{i}^{-1}, x_{1} \ldots x_{m}\right]=\mathbf{1}, i=1, \ldots, m$, принадлежат множеству соотношений копредставления $(22)$, так как $\alpha(s)=\Delta_{m}^{2}$.

Обозначим через $D_{k, m}, k \leqslant m$, полное множество сопряженных элементам $a_{1}$ и $\Delta_{k}^{2}$ в $\mathrm{Br}_{m}$. Положим $\mathscr{D}_{k, m}=S\left(\mathrm{Br}_{m}, D_{k, m}\right)$.

Teорема 3.6. Пусть $C$-группа $G$ принадлежит $\mathscr{C}_{\Delta^{2}, m}$. Тогда существуют число $M \in \mathbb{N}$ и элемент $s \in \mathscr{D}_{m, M}$ такие, что:

(i) $\alpha(s)=\Delta_{M}^{2}$;

(ii) группы $G$ и $G(s)$ являются $C$-изоморфнымми.

ДокАЗАТЕЛЬСТво. Пусть

$$
G=\left\langle x_{1}, \ldots, x_{m} \mid x_{i}^{-1} w_{i, j, k}(\bar{x}) x_{j} w_{i, j, k}(\bar{x})^{-1}=\mathbf{1}, w_{i, j, k}(\bar{x}) \in W\right\rangle
$$

для некоторого конечного множества $W \subset \mathbb{F}_{m}$ такого, что слова $\left[x_{i}^{-1}, x_{1} \ldots x_{m}\right]$ принадлежат множеству $W$ для всех $i=1, \ldots, m$.

Перенумеруем слова $w \in W$ так, чтобы слова $\left[x_{i}^{-1}, x_{1} \ldots x_{m}\right]$ имели номер $i$. Положим $G_{n}=\mathbb{F}_{m} / N_{n}$, где $N_{n}$ - нормальное замыкание множества слов $\bar{W}_{n}=$ $\left\{w_{1}, \ldots, w_{n}\right\}$ в $\mathbb{F}_{m}$. Покажем, что для каждого $n \geqslant m$ найдется элемент $s_{n} \in$ $\mathscr{D}_{m, M_{n}}$ такой, что:

1) $\alpha\left(s_{n}\right)=\Delta_{M_{n}}^{2}$

2) группы $G_{n}$ и $G\left(s_{n}\right)$ являются $C$-изоморфными.

Для $M_{m}=m$ положим $s_{m}=\Delta_{m}^{2} \in \mathscr{D}_{m, m}$. Согласно утверждению 3.5 групшы $G_{m}$ и $G\left(s_{m}\right)$ являются $C$-изоморфными. Предположим, что для некоторых $k \geqslant 0$ и $p \geqslant 0$ уже найден элемент $s_{m+k} \in \mathscr{D}_{m, M_{m+k}}$, где $M_{m+k}=2^{p} m$, такой, что:

1) $\alpha\left(s_{m+k}\right)=\Delta_{M_{m+k}}^{2}$;

2) групшы $G_{m+k}$ и $G\left(s_{m+k}\right)$ являются $C$-изоморфннцми;

3 ) множество соотношений $R_{W_{s_{m+k}}}$ влечет соотношения $R_{\bmod m}$.

Построим элемент $s_{m+k+1}$, имеюший свойства, аналогичные тем, которые имеет элемент $s_{m+k}$. Положим $n=m+k$ и рассмотрим слово

$$
w_{n+1}=x_{i_{n}}^{-1} w_{i_{n}, j_{n}, k_{n}}(\bar{x}) x_{j_{n}} w_{i_{n}, j_{n}, k_{n}}(\bar{x})^{-1} .
$$

Пусть $l$ - буквенная длина слова $w_{i_{n}, j_{n}, k_{n}}$. Рассмотрим элемент $\tilde{s}_{n}=d^{l+2}\left(s_{n}\right)$. Согласно утверждению 1.13 множества соотношений $R_{W_{s_{n}}}$ и $R_{W_{d^{l+2}\left(s_{n}\right)}}$ яВляются эквивалентными. Заметим, что $\alpha\left(d^{l+2}\left(s_{n}\right)\right)=\Delta_{2^{l+2} M_{n}}^{2}$ и условие 3$)$ также выполнено для элемента $\tilde{s}_{n}=d^{l+2}\left(s_{n}\right)$. Положим

$$
y_{n}=\mathrm{m}_{2^{l} M_{n}, m}\left(w_{i_{n}, j_{n}, k_{n}}\right) x_{2^{l} M_{n}+j_{n}} \mathrm{~m}_{2^{l} M_{n}, m}\left(w_{i_{n}, j_{n}, k_{n}}\right)^{-1} .
$$


Согласно утверждениям 1.7 и 1.11 найдется элемент $b \in \mathrm{Br}_{2^{l+2} M_{n}}$, сопряженный стандартной образуюшей $a_{1}$ и такой, что множество соотношений $R\left(W_{\langle b\rangle, \mathbb{F}_{2 l+2} M_{n}}\right)$ эквивалентно одному соотношению $x_{i_{n}+2^{l+1} M_{n}}=y_{n}$. Из леммы 3.4 , (i) следует, что элемент

$$
s_{n+1}=d\left(\lambda_{S}(b)\left(d\left(\tilde{s}_{n}\right)\right), d\left(\tilde{s}_{n}\right), d\left(\tilde{s}_{n}\right), d\left(\tilde{s}_{n}\right)\right)
$$

является искомым, что завершает доказательство теоремы.

\section{§4. Слабая $\mu$-эквивалентность}

Обозначим через $A_{i}=A_{i}(m)$ полное множество сопряженных элементу $a_{1}^{i+1}$ в группе $\mathrm{Br}_{m}$, где $\left\{a_{1}, \ldots, a_{m-1}\right\}$ - множество стандартных образующих группы $\mathrm{Br}_{m}$ (напомним, что все образующие $a_{1}, \ldots, a_{m-1}$ сопряжены друг другу в группе кос $\left.\mathrm{Br}_{m}\right)$. Положим $\mathscr{A}_{k}=S\left(\mathrm{Br}_{m}, A_{\leqslant k}\right), \mathscr{A}_{k}^{0}=S\left(\mathrm{Br}_{m}, A_{\leqslant k}^{0}\right)$, где $A_{\leqslant k}=$ $A_{-3} \cup\left(\bigcup_{i=-1}^{k} A_{i}\right)$ и $A_{\leqslant k}^{0}=\bigcup_{i=0}^{k} A_{i}$. Имеем вложение $\mathscr{A}_{k}^{0} \subset \mathscr{A}_{k}$.

Далее мы ограничимся рассмотрением случая $k=2$. Полугруппа $\mathscr{A}_{2}^{0}$ называется полугруппой каспидальных разложений на множители, а $\mathscr{A}_{2}$ - полугруппой каспидальных разложений на множители с отрицательными ноудами.

Пусть $U \subset \mathrm{Br}_{m}$ - полное множество сопряженных элементов, содержащее $A_{1} \cup A_{-3}$. Заметим, что если $g \in A_{1}$, то $g^{-1} \in A_{-3}$. Рассмотрим полугруппу

$$
\bar{S}_{U}=\langle u \in U \mid R \in \mathscr{R} \cup \overline{\mathscr{R}}\rangle
$$

где $\mathscr{R}$ - множество соотношений, определяющих полугруппу $S_{U}$ (см. $\left.\S 3\right)$, и

$$
\overline{\mathscr{R}}=\left\{g \cdot\left(g^{-1}\right)=\left(g^{-1}\right) \cdot g=\mathbf{1} \mid g \in A_{1}\right\}
$$

- множество соотношений сокращения. Существует канонический гомоморфизм полугрупा

$$
c: \mathscr{S}_{U} \rightarrow \bar{S}_{U}
$$

Скажем, что два элемента $s_{1}, s_{2} \in S_{U}$ являются слабо әквивалентными, если $c\left(s_{1}\right)=c\left(s_{2}\right)$. Легко видеть, что полугруппа $\bar{S}_{U}$ может быть рассмотрена как полугруппа над $\mathrm{Br}_{m}$ с гомоморфизмом произведения $\bar{\alpha}: \bar{S}_{U} \rightarrow \mathrm{Br}_{m}$ таким, что $\alpha=\bar{\alpha} \circ c$.

Пусть $\left(s, \mu_{s}\right)$ - пара, состоящая из элемента $s \in S_{U}$ и гомоморфизма $\mu_{s}$ : $G(s) \rightarrow \mathfrak{S}_{N}$, где $\mathfrak{S}_{N}$ - симметрическая группа для некоторого $N$. Скажем, что две пары $\left(s_{1}, \mu_{s_{1}}\right)$ и $\left(s_{2}, \mu_{s_{2}}\right)$ являются әквивалентнылми, если $s_{1}$ и $s_{2}$ принадлежат одной и той же орбите при действии сопряжением группы $\mathrm{Br}_{m}$ на $S_{U}$ (т. е. сушествует элемент $b \in \mathrm{Br}_{m}$ такой, что $\left.s_{2}=\lambda(b)\left(s_{1}\right)\right)$ и $\mu_{2}=\mu_{1} \circ \gamma_{b}$, где $\gamma_{b}-$ изоморфизм, определенный в утверждении 3.1 .

Говорят, что две пары $\left(s_{1}, \mu_{s_{1}}\right)$ и $\left(s_{2}, \mu_{s_{2}}\right)$ получены друг из друга с помощью допустимого преобразования $\left(s_{1}, \mu_{s_{1}}\right) \leftrightarrow\left(s_{2}, \mu_{s_{2}}\right)$, если существует такой элемент $g \in A_{1}$, что $s_{2}=g \cdot\left(g^{-1}\right) \cdot s_{1}, \quad \mu_{1}=\psi_{g \cdot\left(g^{-1}\right)} \circ \mu_{2}$ и порядок групшы $\mu_{s_{2}}\left(G_{g, \text { lос }}\right)$ больше двух. Скажем, что две пары $\left(s^{\prime}, \mu_{s^{\prime}}\right)$ и $\left(s^{\prime \prime}, \mu_{s^{\prime \prime}}\right)$ являются слабо $\mu$-эквивалентными, если существует последовательность пар $\left(s_{1}, \mu_{1}\right), \ldots$ $\ldots,\left(s_{n}, \mu_{n}\right)$ такая, что $\left(s^{\prime}, \mu_{s^{\prime}}\right)=\left(s_{1}, \mu_{1}\right),\left(s^{\prime \prime}, \mu_{s^{\prime \prime}}\right)=\left(s_{n}, \mu_{n}\right)$ и для каждого 
$i=1, \ldots, n-1$ пары $\left(s_{i}, \mu_{i}\right)$ и $\left(s_{i+1}, \mu_{i+1}\right)$ либо эквивалентны, либо получены друг из друга с помошью допустимого преобразования.

Ниже мы рассмотрим случай, когда $U=\mathscr{A}_{2}$. Пусть $s=u_{1} \cdot \ldots \cdot u_{n} \in \mathscr{A}_{2}$, $u_{i} \in A_{\leqslant 2}$. Скажем, что гомоморфизм $\mu_{s}: G(s) \rightarrow \mathfrak{S}_{N}$ является общ, им, если:

(i) $\mu_{s}$ является эпиморфизмом;

(ii) $\mu_{s}\left(\varphi_{s}\left(x_{i}\right)\right)$ является транспозицией в симметрической группе $\mathfrak{S}_{N}$ для каждой $C$-образующей $\varphi_{s}\left(x_{i}\right)$ групшы $G(s)$;

(iii) группа $\mu_{s}\left(G_{u_{i}, \text { loc }}\right)$ имеет порядок больше двух для каждого множителя $u_{i} \in A_{-3} \cup A_{1} \cup A_{2}$.

Пусть $\left(s, \mu_{s}\right)$ - пара, состояшая из элемента $s \in \mathscr{A}_{2}$ и гомоморфизма $\mu_{s}$ : $G(s) \rightarrow \mathfrak{S}_{N}$ для некоторого $N$. Согласно утверждению 3.3 существует канонический $C$-изоморфизм $\psi_{d}: G(d(s)) \rightarrow G(s)$. Он определяет пару $\left(d(s), d\left(\mu_{s}\right)\right)$, где $d\left(\mu_{s}\right)=\mu_{s} \circ \psi_{d}$.

УТВЕРЖДЕНИЕ 4.1. Пусть $s \in \mathscr{A}_{2} u \mu_{s}: G(s) \rightarrow \mathfrak{S}_{N}-$ общий эпиморфизм. Тогда гомоморфизм $d\left(\mu_{s}\right): G(d(s)) \rightarrow \mathfrak{S}_{N}$ является общим.

ДОКАЗАТЕЛЬСТвО проводится прямой проверкой.

Tеорема 4.2. Пусть әлемент $s=u_{1} \cdot \ldots \cdot u_{n} \in \mathscr{A}_{2} \subset S_{\mathrm{Br}_{m}}, u_{i} \in A_{\leqslant 2}$, такой, что $\alpha(s)=\Delta_{m}^{2}$, и пусть $\mu_{s}: G(s) \rightarrow \mathfrak{S}_{N}-$ общий эпиморфизм. Пусть образующая $x_{i}$ группы $\mathbb{F}_{m}$ и әлемент $y$, сопряженный в $\mathbb{F}_{m}$ некоторой образующей $x_{j}$, таковь, что $\mu_{s}\left(\varphi_{s}\left(x_{i}\right)\right)$ и $\mu_{s}\left(\varphi_{s}(y)\right)$ - несовпадающие коммутирующие транспозичии в $\mathfrak{S}_{N}$. Тогда существуют иисло $M \in \mathbb{N}$ и две пары $\left(\bar{s}, \mu_{\bar{s}}\right) u\left(\tilde{s}, \mu_{\tilde{s}}\right), \bar{s}, \tilde{s} \in \mathscr{A}_{2} \subset S_{\mathrm{Br}_{M}}$, такие, ито:

(i) $\alpha(\bar{s})=\alpha(\tilde{s})=\Delta_{M}^{2}$;

(ii) $C$-группа $G_{\bar{s}}$ является $C$-изоморфной $C$-группе $G(s)$, а $C$-группа $G_{\tilde{s}}$ является $C$-изоморфной $C$-группе

$$
G(s) /\left\{\left[\varphi_{s}\left(x_{i}\right), \varphi_{s}(y)\right]=\mathbf{1}\right\}
$$

(iii) если $\left[\varphi_{s}\left(x_{i}\right), \varphi_{s}(y)\right] \neq \mathbf{1}$, то типь әлементов $\bar{s}$ и $\tilde{s}$ различны;

(iv) $\mu_{\bar{s}}$ и $\mu_{\tilde{s}}$ являются общими әпиморфизмами в симметрическую груп$n y \mathfrak{S}_{N}$

(v) пары $\left(\bar{s}, \mu_{\bar{s}}\right)$ и $\left(\tilde{s}, \mu_{\tilde{s}}\right)$ слабо $\mu$-эквивалентны.

Более того, если $s \in \mathscr{A}_{2}^{0} \subset S_{\mathrm{Br}_{m}}$, то $\bar{s}, \tilde{s} \in \mathscr{A}_{2}^{0} \subset S_{\mathrm{Br}_{M}}$.

ДоКАЗАТЕЛЬСТво. Согласно утверждениям 1.7, 1.8, 1.11 и 4.1 сушествуют:

1) число $M_{1}=2^{n} m$ (для некоторого $n \in \mathbb{N}$ );

$2)$ элемент $y_{1} \in \mathbb{F}_{M_{1}}$, являющийся раздвижкой элемента $y$ и такой, что $\left(y_{1}, x_{k}\right)$ хорошая пара для некоторого $k \equiv i \bmod m$;

3) элемент $s_{1}=d^{n}(s) \in \mathscr{A}_{2} \subset S_{\mathrm{Br}_{M_{1}}}$ такой, что $\mu_{s}\left(x_{i}\right)=\mu_{s_{1}}\left(x_{k}\right), \mu_{s}(y)=$ $\mu_{s_{1}}\left(y_{1}\right)$, где $\mu_{s_{1}}=d^{n}\left(\mu_{s}\right)$.

По определению хороших пар найдется элемент $g \in \mathrm{Br}_{M_{1}}$ такой, что $g\left(x_{1}\right)=y_{1}$ и $g\left(x_{k}\right)=x_{2}$. Положим $b=g a_{1} g^{-1} \in \mathrm{Br}_{M_{1}}, \bar{s}=d^{2}\left(s_{1}\right)$ и $\tilde{s}=d\left(\lambda_{S}\left(b^{2}\right)(d(s)), d(s)\right.$, $d(s), d(s))$. Согласно утверждению 4.1 группы $G(s)$ и $G(\bar{s})$ канонически $C$-изоморфны. Следовательно, общий эпиморфизм $\mu_{s}$ индуцирует общий эпиморфизм 
$\mu_{\bar{s}}: G(\bar{s}) \rightarrow \mathfrak{S}_{N}$. Из доказательства леммы 3.4 ,(ii) следует, что групшы $G(\tilde{s})$ и $G\left(b^{-2} \cdot b^{2} \cdot \bar{s}\right)$ канонически $C$-изоморфин группе

$$
G(\bar{s}) / N_{G(\bar{s})}\left(\varphi_{W_{\bar{s}}}\left(\left[x_{j}, y_{1}\right]\right)\right),
$$

где $\tilde{s}=d\left(\lambda_{S}\left(b^{2}\right)\left(d\left(s_{1}\right)\right), d\left(s_{1}\right), d\left(s_{1}\right), d\left(s_{1}\right)\right)$. Поэтому эти изоморфизмы индуцируют обшие эпиморфизмы

$$
\mu_{b^{-2} \cdot b^{2} \cdot \bar{s}}: G\left(b^{-2} \cdot b^{2} \cdot \bar{s}\right) \rightarrow \mathfrak{S}_{N} \quad \text { и } \quad \mu_{\tilde{s}}: G(\tilde{s}) \rightarrow \mathfrak{S}_{N} .
$$

Имеем $\left(\bar{s}, \mu_{\bar{s}}\right) \leftrightarrow\left(b^{-2} \cdot b^{2} \cdot \bar{s}, \mu_{b^{-2} \cdot b^{2} \cdot \bar{s}}\right)$, пары $\left(b^{-2} \cdot b^{2} \cdot \bar{s}, \mu_{b^{-2} \cdot b^{2} \cdot \bar{s}}\right)$ и $\left(b^{-2} \cdot b^{2} \cdot \tilde{s}\right.$, $\left.\mu_{b^{-2} \cdot b^{2} \cdot \tilde{s}}\right)$ являются эквивалентными и $\left(b^{-2} \cdot b^{2} \cdot \tilde{s}, \mu_{b^{-2} \cdot b^{2} \cdot \tilde{s}}\right) \leftrightarrow\left(\tilde{s}, \mu_{\tilde{s}}\right)$. Следовательно, пары $\left(\bar{s}, \mu_{\bar{s}}\right)$ и $\left(\tilde{s}, \mu_{\tilde{s}}\right)$ слабо $\mu$-эквивалентны, что завершает доказательство теоремы.

\section{§5. Кривые Гурвица}

Пусть $F_{N}$ - относительно минимальная линейчатая поверхность, $N \geqslant 1$, pr: $F_{N} \rightarrow \mathbb{C P}^{1}$ - ее линейчатая структура, $R$ - слой проекции pr и $E_{N}-$ исключительное сечение, $E_{N}^{2}=-N$. В дальнейшем pr: $F_{1} \rightarrow \mathbb{C P}^{1}$ будем отождествлять с линейной проекцией $\mathrm{pr}: \mathbb{C P}^{2} \rightarrow \mathbb{C P}^{1}$ из точки $p \in \mathbb{C P}^{2}(p-$ образ при стягивании кривой $E_{1}$ в точку).

ОпРЕДЕЛЕНИЕ 5.1. Образ $\bar{H}=f(S) \subset F_{N}$ гладкого отображения $f: S \rightarrow$ $F_{N} \backslash E_{N}$ ориентированной замкнутой вешественной поверхности $S$ называется $к p u$ вой Гурвица (относительно проекиии pr) степени $m$, если сушествует конечное подмножество $Z \subset \bar{H}$ такое, что:

(i) $f$ является вложением поверхности $S \backslash f^{-1}(Z)$ и для любого $s \notin Z$ образ Гурвица $\bar{H}$ и слой $R_{\operatorname{pr}(s)}$ проекции $\operatorname{pr}$ пересекаются в $s$ трансверсально с положительным индексом пересечения;

(ii) для каждой точки $s \in Z$ существует окрестность $U \subset F_{N}$ такая, что $\bar{H} \cap U$ является комплексно-аналитической кривой и комплексная ориентация на $\bar{H} \cap U \backslash\{s\}$ совпадает с ориентацией, индуцированной ориентацией на $S$ с помощью $f$;

(iii) ограничение pr на $\bar{H}$ является конечным отображением степени $m$.

Для каждой кривой Гурвица $\bar{H}$ существует единственное минимальное множество $Z \subset \bar{H}$, удовлетворяющее условиям определения 5.1. Обозначим его через $Z(\bar{H})$.

Кривая Гурвица $\bar{H}$ называется каспидальной, если для каждой точки $s \in Z(\bar{H})$ найдутся окрестность $U$ и локальные аналитические координаты $z, w$ в $U$ такие, что:

(iv) проекция $\operatorname{pr}_{\mid U}$ совпадает с отображением $(z, w) \mapsto z$;

(v) $\bar{H} \cap U$ задана уравнением $w^{2}=z^{k}, k \geqslant 1$.

Каспидальная кривая называется обыкновенной каспидальной, если в условии (v) имеет место неравенство $k \leqslant 3$ для всех $s \in Z(\bar{H})$, и нодальной, если $k \leqslant 2$. Кроме того, далее будем рассматривать также (обыкновенные) каспидальные кривые Гурвица $\bar{H}$ с отрицательнымми ноудами, т.е. $\bar{H}$ могут иметь еще и особые точки следующего вида: 
(vi) в некоторой окрестности $U \subset F_{N}$ точки $s \in \bar{H}$ пересечение $U \cap \bar{H}$ распадается на две ветви, пересекающиеся трансверсально в $s$ с отрицательным индексом пересечения и пересекающие слой $R_{\operatorname{pr}(s)}$ трансверсально с положительным индексом пересечения.

Поскольку $E_{N} \cap \bar{H}=\varnothing$, то можно определить брэйд-монодромное разложение на множители $\operatorname{bmf}(\bar{H}) \in S_{\mathrm{Br}_{m}}$ кривой Гурвица $\bar{H}$ аналогично тому, как это было сделано в алгебраическом случае (см., например, [7]). Чтобы определить $\mathrm{bmf}(\bar{H})$, зафиксируем слой $R_{\infty}$, пересекающий трансверсально кривую $\bar{H}$, и рассмотрим аффинную кривую Гурвица $H=\bar{H} \cap \mathbb{C}^{2}$, где $\mathbb{C}^{2}=F_{N} \backslash\left(R_{\infty} \cup E_{N}\right)$. Выберем $r \gg 1$ так, что $\operatorname{pr}(Z)$ содержится в диске $D(r)=\{|z| \leqslant r\} \subset \mathbb{C}=\mathbb{C P}^{1} \backslash \operatorname{pr}\left(F_{\infty}\right)$, $Z=Z(\bar{H})$. Обозначим через $z_{1}, \ldots, z_{n}$ элементы из $\operatorname{pr}(Z)$. Выберем $\varepsilon, 0<\varepsilon \ll 1$, такое, что диски $D_{i}(\varepsilon)=\left\{z \in \mathbb{C}|| z-z_{i} \mid \leqslant \varepsilon\right\}, i=1, \ldots, n$, не пересекаются друг с другом. Выберем также произвольные точки $u_{i} \in \partial D_{i}(\varepsilon)$, точку $u_{0} \in \partial D(r)$ и непересекаюшиеся простые пути $l_{i} \subset D(r) \backslash \bigcup_{j=1}^{n} D_{j}(r), i=1, \ldots, n$, с началом в точке $u_{0}$ и концами в точках $u_{i}$. Перенумеруем выбранные точки таким образом, чтобы произведение $\gamma_{1} \ldots \gamma_{n}$ петель $\gamma_{i}=l_{i} \circ \partial D_{i}(\varepsilon) \circ l_{i}^{-1}$ стало равно $\partial D(r)$ в $\pi_{1}\left(D(r) \backslash\left\{z_{1}, \ldots, z_{n}\right\}, u_{0}\right)$.

Каждая петля $\gamma_{i}$, поднятая на $H$, определяет элемент $b_{i} \in \mathrm{Br}_{m}$. Разложение $b_{1} \cdot \ldots \cdot b_{n} \in S_{\mathrm{Br}_{m}}$ называется разложением на множители брэйд-монодромии кривой Гурвица $\bar{H}$. Фактически, каждый $b_{i}$ сопряжен стандартной форме брэйдмонодромии ростка некоторой алгебраической особенности над точкой $z_{i}$, либо $b_{i} \in A_{-3}$, если соответствуюшая особая точка кривой $\bar{H}$ является отрицательным ноудом. Следовательно, $\operatorname{bmf}(\bar{H})=b_{1} \cdot \ldots \cdot b_{n}$ принадлежит полугруппе $\overline{\mathscr{P}}=S\left(\mathrm{Br}_{m}, P \cup A_{-3}\right)$, где $P$ - множество брэйд-монодромий всех ростков алгебраических кривых степени $m$. Орбита элемента $\operatorname{bmf}(\bar{H})$ при действии сопряжением группы $\mathrm{Br}_{m}$ на полугруппе $\bar{P}$ называется типом брәйд-монодромного разложения на множители кривой $\bar{H}$ и обозначается через $\operatorname{bmt}(\bar{H})$.

Если $\bar{H}$ - обыкновенная каспидальная кривая Гурвища с отрицательными ноудами, то ее разложение на множители брэйд-монодромии $\operatorname{bmf}(\bar{H}) \in \mathscr{A}_{2}$.

Следующая лемма является хорошо известной (см., например, [7]).

Лемма 5.2. Для кривой Гурвица $\bar{H} \subset F_{N}$ степени $m$ имеем

$$
\alpha(\operatorname{bmf}(\bar{H}))=\Delta_{m}^{2 N}
$$

Так же можно непосредственно доказать обратное утверждение.

Tеорема 5.3 [15]. Для кажсдого $b=b_{1} \cdot \ldots \cdot b_{n} \in \overline{\mathscr{P}}$ maкого, что $\alpha(b)=\Delta_{m}^{2 N}$, существует кривая Гурвица $\bar{H} \subset F_{N}$, имеющая брэйд-монодромное разложение на множители $\operatorname{bmf}(\bar{H})$, равное $b$.

ОПРЕДЕЛЕНИЕ 5.4. Две кривые Гурвица $\bar{H}_{1}, \bar{H}_{2} \subset F_{N}$ называются $H$-изотопнылми, если сушествует послойная непрерывная изотопия $\varphi_{t}: F_{N} \rightarrow F_{N}$, $t \in[0,1]$, гладкая вне слоев $R_{\mathrm{pr}(s)}, s \in Z\left(\bar{H}_{1}\right)$, и такая, что:

(i) $\varphi_{0}=\mathrm{Id}$;

(ii) $\varphi_{t}\left(\bar{H}_{1}\right)$ является кривой Гурвица для всех $t \in[0,1]$;

(iii) $\varphi_{1}\left(\bar{H}_{1}\right)=\bar{H}_{2}$;

(iv) $\varphi_{t}=\mathrm{Id}$ в окрестности кривой $E_{N}$ для всех $t \in[0,1]$. 
Согласно [7, теорема 3.2] две каспидальные кривые Гурвица с отрицательными ноудами являются $H$-изотопными тогда и только тогда, когда они имеют одинаковый брэйд-монодромный тип разложения на множители.

Очевидно, если $\bar{H}_{1}$ и $\bar{H}_{2}$ являются $H$-изотопными, то фундаментальные группы $\pi_{1}\left(F_{N} \backslash \bar{H}_{1}\right)$ и $\pi_{1}\left(F_{N} \backslash \bar{H}_{2}\right)$ изоморфны.

Рассмотрим два сечения $H_{1}=\{u+1=0, v=0\}$ и $H_{2}=\{u=0, v=0\}$ проекции $\operatorname{pr}_{1}: D \rightarrow D_{1}$ бидиска $D=D_{1} \times D_{2}$, где $D_{1}=\left\{\sqrt{x^{2}+y^{2}} \leqslant 2\right\}$ и $D_{2}=\left\{\sqrt{u^{2}+v^{2}} \leqslant 2\right\}$. Пусть $h_{t}-$ изотопия сечения $H_{1}$, заданная уравнениями

$$
\begin{gathered}
u+1+2 t \rho\left(\sqrt{x^{2}+y^{2}}\right)\left(y+x^{2}-1\right)=0, \\
v-t \lambda \rho\left(\sqrt{x^{2}+y^{2}}\right) y=0,
\end{gathered}
$$

где $\rho: \mathbb{R} \rightarrow \mathbb{R}$-монотонная $C^{\infty}$-функция такая, что $\rho(s)=0$, если $s \geqslant 2$, и $\rho(s)=1$, если $s \leqslant 1$, а $\lambda$ - малая положительная константа.

Легко видеть, что:

1) $h_{t}\left(H_{1}\right), 0 \leqslant t \leqslant 1$, и $H_{2}$ являются симплектическими поверхностями в $D$ относительно симплектической формы $\omega=x \wedge y+u \wedge v$;

2 ) сечения $h_{t}\left(H_{1}\right)$ и $H_{2}$ не имеют обших точек, если $0 \leqslant t<1 / 2$;

$3)$ сечение $h_{t}\left(H_{1}\right)$ пересекает $H_{2}$ трансверсально в двух точках, соответственно с положительным и отрицательным индексом пересечения, если $1 / 2<t \leqslant 1$.

Назовем "регулярную гомотопию" $h_{t}\left(H_{1}\right) \cup H_{2}$ рождением отрицательного ноуда. Обратная гомотопия называется уничтожением отрицательного ноуда.

Скажем, что две кривые Гурвица $\bar{H}^{\prime}, \bar{H}^{\prime \prime} \subset F_{N}$ получены друг из друга с помощью допустимого преобразования, если в некоторой окрестности $U \subset F_{N}$, диффеоморфной бидиску $D=D_{1} \times D_{2}$ с локальными аналитическими координатами $z=x+i y$ и $w=u+i v$ такими, что $\operatorname{pr}_{\mid U}=\operatorname{pr}_{1}: D \rightarrow D_{1}$, имеем

$$
\begin{gathered}
\bar{H}^{\prime} \cap U=\{u+1=0, v=0\} \cup\{u=0, v=0\}, \\
\bar{H}^{\prime \prime} \cap U=\left\{u+1+2\left(y+x^{2}-1\right) \rho=0, v-\rho y=0\right\} \cup\{u=0, v=0\}
\end{gathered}
$$

и $\bar{H}^{\prime} \cap\left(F_{N} \backslash U\right)=\bar{H}^{\prime \prime} \cap\left(F_{N} \backslash U\right)$.

Легко видеть, что если $\bar{H}^{\prime}, \bar{H}^{\prime \prime} \subset F_{N}$ получены друг из друга с помошью допустимого преобразования, то

$$
\operatorname{bmf}\left(\bar{H}^{\prime \prime}\right)=g \cdot\left(g^{-1}\right) \cdot \operatorname{bmf}\left(\bar{H}^{\prime}\right)
$$

для некоторого $g \in A_{1}$.

Скажем, что $\bar{H}^{\prime}, \bar{H}^{\prime \prime} \subset F_{N}$ являются слабо әквивалентнымии, если существует такая последовательность кривых Гурвица $\bar{H}_{i}, i=1, \ldots, n$, что $\bar{H}^{\prime}=\bar{H}_{1}$, $\bar{H}^{\prime \prime}=\bar{H}_{n}$ и для каждого $i=1, \ldots, n-1$ кривые Гурвица $\bar{H}_{i}, \bar{H}_{i+1}$ либо $H$-изотопны, либо получены друг из друга с помощью допустимого преобразования.

УтвеРжДЕНИЕ 5.5. Две каспидальные кривые Гурвица $\bar{H}^{\prime}, \bar{H}^{\prime \prime} \subset F_{N}$ (возможно, с отрицательными ноудами) являются слабо әквивалентныцми тогда и только тогда, когда их типь разложения на множители брәйд-монодромии $\operatorname{bmf}\left(\bar{H}^{\prime}\right) u \operatorname{bmf}\left(\bar{H}^{\prime \prime}\right)$ являются слабо әквивалентными. 
ДоКАЗАТЕЛЬСТво следует из равенства (26) и [7, теорема 3.2].

Отметим, что если две каспидальные кривые Гурвица $\bar{H}^{\prime}, \bar{H}^{\prime \prime} \subset F_{N}$ слабо эквивалентны, то не обязательно их фундаментальные группы $\pi_{1}\left(F_{N} \backslash \bar{H}^{\prime}\right)$ и $\pi_{1}\left(F_{N} \backslash \bar{H}^{\prime \prime}\right)$ являются изоморфными.

\section{§6. Фундаментальные группы дополнений к кривым Гурвица}

Следующая теорема является хорошо известной.

Tеорема (Зариского-ван Кампена). Пусть $\bar{H} \subset F_{N}-$ кривая Гурвица степени $m$, имеющая разложение на множители брәйд-монодромии $\operatorname{bmf}(\bar{H})=s$. Тогда:

(i) $\pi_{1}\left(\mathbb{C}^{2} \backslash \bar{H}\right) \simeq G\left(B_{s}\right)$, зде $\mathbb{C}^{2}=F_{N} \backslash\left(E_{N} \cup R_{\infty}\right)$;

(ii) $\pi_{1}\left(\mathbb{C P}^{2} \backslash \bar{H}\right) \simeq G\left(B_{s}\right) /\left\{\varphi_{W_{s}}\left(x_{1} \ldots x_{m}\right)=\mathbf{1}\right\}$.

В случае $N=1$, так как $\alpha(s)=\Delta_{m}^{2}$ для $s=\operatorname{bmf}(\bar{H})$, получаем, что соотношения

$$
\left[x_{i}^{-1}, x_{1} \ldots x_{m}\right]=\mathbf{1}, \quad i=1, \ldots, m,
$$

принадлежат множеству определяюших соотношений копредставления (22).

Обозначим через $\mathscr{H}$ подкласс в $\mathscr{C}$, состоящий из фундаментальных групп дополнений аффинных плоских кривых Гурвица, т. е.

$$
\mathscr{H}=\left\{\pi_{1}\left(\mathbb{C P}^{2} \backslash\left(\bar{H} \cup R_{\infty}\right)\right)\right\}
$$

где $R_{\infty}$ - общая прямая пучка, определяющего линейчатую структуру $\mathrm{pr}: \mathbb{C P}^{2} \rightarrow$ $\mathbb{C P}^{1}$ на $\mathbb{C P}^{2}$.

Tеорема 6.1. Имеем $\mathscr{H}=\mathscr{C}_{\Delta^{2}}$.

ДокАЗАТЕЛЬСтво. Вложение $\mathscr{H} \subset \mathscr{C}_{\Delta^{2}}$ следует из формул $(22),(27)$ и утверждения 1.6.

Обратное вложение вытекает из следующей теоремы.

Теорема 6.2. Для каждой $C$-группы $G \in \mathscr{C}_{\Delta^{2}, m}$ существует кривая Гурвица $\bar{H} \subset \mathbb{C P}^{2}$ с особьми точками типа $\left\{w^{m}=z^{m}\right\}$ такая, ито $\pi_{1}\left(\mathbb{C}^{2} \backslash H\right)$ $C$-изоморфна әруппе $G$, әде $\mathbb{C}^{2}=\mathbb{C P}^{2} \backslash R_{\infty} u H=\bar{H} \cap \mathbb{C}^{2}$.

ДокАЗАТЕЛЬСТво. Брэйд-монодромия особой точки $\left\{w^{m}=z^{m}\right\}$ равна $\Delta_{m}^{2}$. Поэтому теорема следует из теорем 3.6 и 5.3.

Обозначим через $\mathscr{T}$ подкласс в $\mathscr{K}$, состояший из групп торических узлов, т. е. фундаментальных групп $\pi_{1}\left(S^{3} \backslash K_{p, q}\right)$ узлов

$$
K_{p, q}=\left\{(z, w) \in S^{3} \mid z=e^{2 p \pi i t}, w=e^{2 q \pi i t}, t \in[0,1]\right\}
$$

$(p, q-$ взаимно простые числа, возможно, $p=q=1)$, где

$$
S^{3}=\left\{\left.(z, w) \in \mathbb{C}^{2}|| z\right|^{2}+|w|^{2}=2\right\} .
$$

Tеорема 6.3. Имеем $\mathscr{H} \cap \mathscr{K}=\mathscr{T}$. 
ДокАЗАТельство. Пусть $\bar{H} \subset \mathbb{C P}^{2}-$ кривая Гурвища степени $m$ и $G=$ $\pi_{1}\left(\mathbb{C}^{2} \backslash H\right)$ - фундаментальная группа дополнения к ее аффинной части $H \subset \mathbb{C}^{2}=$ $\mathbb{C P}^{2} \backslash R_{\infty}$. Из соотношений (27) следует, что элемент $\varphi_{W_{s}}\left(x_{1} \ldots x_{m}\right)$ принадлежит центру группы $G$. Поэтому из $[4$, теорема 6.1$]$ следует, что $\mathscr{H} \cap \mathscr{K} \subset \mathscr{T}$.

Группа $G_{p, q}$ узла $K_{p, q}$ имеет копредставление Виртингера

$$
G_{p, q}=\left\langle x_{1}, \ldots, x_{p} \mid x_{i}=\left(a_{1} \ldots a_{p-1}\right)^{q}\left(x_{i}\right), i=1, \ldots, p\right\rangle .
$$

Имеем

$$
\left(a_{1} \ldots a_{p-1}\right)^{p q}=\Delta_{p}^{2 q} .
$$

Следовательно, существует кривая Гурвица $\widetilde{H} \subset F_{q}$ степени $p$ с разложением на множители брэйд-монодромии $\operatorname{bmf}(\widetilde{H})=s^{p} \in S_{\mathrm{Br}_{p}}$, где $s=\left(a_{1} \ldots a_{p-1}\right)^{q}-$ одна из образуюших полугруппы $S_{\mathrm{Br}_{p}}$ ( $s$ является брэйд-монодромией особой точки, заданной уравнением $\left.w^{p}=z^{q}\right)$. Согласно теореме Зариского-ван Кампена имеем

$$
\pi_{1}\left(\mathbb{C}^{2} \backslash H\right) \simeq G_{p, q},
$$

где $H=\mathbb{C}^{2} \cap \widetilde{H}, \mathbb{C}^{2}=F_{q} \backslash R_{\infty}$ и $R_{\infty}-$ слой проекции $\mathrm{pr}$, пересекающий $\widetilde{H}$ трансверсально.

Как известно (см., например, [16]),

$$
\Delta_{p+1}^{2}=\prod_{l=p+1}^{2} \prod_{k=1}^{l-1} b_{k, l}^{2}=\Delta_{p}^{2} \prod_{k=1}^{p} b_{k, p+1}^{2},
$$

где $b_{k, l}=\left(a_{l-1} \ldots a_{k+1}\right) a_{k}\left(a_{l-1} \ldots a_{k+1}\right)^{-1}$ для $k<l$ (здесь $\prod_{k}^{n}$ обозначает произведение слева направо от $k$ до $n)$.

Положим

$$
s_{1}=\prod_{k=1}^{p}\left(b_{k, p+1}^{2}\right) \in S_{\mathrm{Br}_{p+1}},
$$

где произведение взято в полугруппе $S_{\mathrm{Br}_{p+1}}$. Элемент $s^{p} \cdot s_{1}^{q} \in S_{\mathrm{Br}_{p+1}}$ является разложением на множители брэйд-монодромии кривой Гурвица в $F_{q}$, так как $\alpha\left(s^{p} \cdot s_{1}^{q}\right)=\Delta_{p+1}^{2 q}$. Легко видеть, что эта кривая распадается на две компоненты, одна из которых $H$-изотопна кривой $\widetilde{H}$, а другая - сечению $\widetilde{C}$, не пересекаюшемуся с $E_{q}, \operatorname{bmf}(\widetilde{H} \cup \widetilde{C})=s^{p} \cdot s_{1}^{q}$. Кривые $\widetilde{H}$ и $\widetilde{C}$ пересекаются трансверсально. Без ограничения общности можно считать, что $\widetilde{C} \subset F_{q}$ является алгебраическим сечением. Из вида разложения на множители брэйд-монодромии $\operatorname{bmf}(\widetilde{H} \cup \widetilde{C})=s^{p} \cdot s_{1}^{q}$ следует, что фундаментальная группа $\pi_{1}\left(\mathbb{C}^{2} \backslash(\widetilde{H} \cup \widetilde{C})\right)$ канонически $C$-изоморфна прямому произведению

$$
G_{p, q} \times \mathbb{F}_{1} \simeq\left\langle x_{1}, \ldots, x_{p} \mid x_{i}=\left(a_{1} \ldots a_{p-1}\right)^{q}\left(x_{i}\right), i=1, \ldots, p\right\rangle \times\left\langle x_{p+1}\right\rangle
$$

(множитель $\mathbb{F}_{1}=\left\langle x_{p+1}\right\rangle$ соответствует фундаментальной группе $\pi_{1}\left(\mathbb{C}^{2} \backslash \widetilde{C}\right)$ ).

Выберем неоднородные координаты $(u, v)$ в $\mathbb{C}^{2}=F_{q} \backslash\left(E_{q} \cup R_{\infty}\right)$ так, что $u=0$ является уравнением кривой $C=\widetilde{C} \cap\left(F_{q} \backslash\left(E_{q} \cup R_{\infty}\right)\right)$, и координаты $(z, w)$ в дополнении к некоторой прямой $L=L_{\infty} \subset \mathbb{C P}^{2}$. Пусть $f: \mathbb{C P}^{2} \rightarrow F_{q}-$ 
циклическое (рациональное) накрытие степени $p$, заданное уравнениями $z=u^{q}$, $w=v$. Оно разветвлено вдоль $\widetilde{C} \cup E_{q}$. Положим $\bar{H}=f^{-1}(\widetilde{H})$ и $\bar{C}=F^{-1}(\widetilde{C})$. Имеем $L_{\infty}=f^{-1}\left(R_{\infty} \cup E_{q}\right), \bar{H}$ - кривая Гурвища степени $p q$ и $\bar{C}-$ прямая в $\mathbb{C P}^{2}$, пересекающая $\bar{H}$ трансверсально. Легко видеть, что $f_{*}$ определяет изоморфизм групп $\pi_{1}\left(\mathbb{C}^{2} \backslash(\bar{H} \cup \bar{C})\right)$ и $G_{p, q} \times\left\langle x_{p+1}^{q}\right\rangle$, где группа $\left\langle x_{p+1}^{q}\right\rangle$ порождена образом обхода вокруг прямой $\bar{C}$. Следовательно, канонический эпиморфизм

$$
\psi: \pi_{1}\left(\mathbb{C}^{2} \backslash(\bar{H} \cup \bar{C})\right) \rightarrow \pi_{1}\left(\mathbb{C}^{2} \backslash \bar{H}\right),
$$

имеюший ядро $\operatorname{ker} \psi=\left\langle x_{p+1}^{q}\right\rangle$, задает изоморфизм между $G_{p, q}$ и $\pi_{1}\left(\mathbb{C}^{2} \backslash \bar{H}\right)$.

\section{§7. Симплектоморфизмы общих накрытий плоскости}

По определению алгебраическое разветвленное накрытие плоскости $\mathbb{C P}^{2}$ - это конечное голоморфное отображение $f: X \rightarrow \mathbb{C P}^{2}$ нормальной проективной неприводимой комплексной поверхности $X$ в проективную плоскость. Дивизор ветвления $R \subset X$ - это дивизор якобиана отображения $f$ (кратность $R$ равна локальной степени отображения $f$ минус 1$)$. Кривая ветвления $\bar{H} \subset \mathbb{C P}^{2}$ - это образ носителя дивизора $R$, или, другими словами, множество точек, над которыми $f$ не является локально обратимым отображением.

Фундаментальная группа $\pi_{1}=\pi_{1}\left(\mathbb{C P}^{2} \backslash \bar{H}, p\right)$ дополнения кривой $\bar{H}$, где $p \in$ $\mathbb{C P}^{2} \backslash \bar{H}$, действует на слое $f^{-1}(p)$. Тем самым определен гомоморфизм (монодромия степени $N=\operatorname{deg} f) \bar{\mu}=\bar{\mu}(f): \pi_{1} \rightarrow \mathfrak{S}_{N}$ из $\pi_{1}$ в симметрическую группу $\mathfrak{S}_{N}$. Монодромия $\bar{\mu}$ определяется отображением $f$ однозначно с точностью до внутренних автоморфизмов симметрической группы. Обратно, согласно теореме Грауерта-Ремерта [6] гомоморфизм $\bar{\mu}: \pi_{1} \rightarrow \mathfrak{S}_{N}$, образ $\operatorname{Im} \bar{\mu}$ которого транзитивно действует на множестве из $N$ элементов, является монодромией некоторого конечного морфизма $f: X \rightarrow \mathbb{C P}^{2}$ степени $\operatorname{deg} f=N$.

Чтобы описать фундаментальную групу дополнения кривой $\bar{H}$ степени $\operatorname{deg} \bar{H}=m$, зафиксируем точку $p \in \mathbb{C P}^{2} \backslash \bar{H}$, выберем точку $x \in \bar{H} \backslash \operatorname{Sing} \bar{H}$ и рассмотрим проективную прямую $\Pi=\mathbb{C P}^{1} \subset \mathbb{C P}^{2}$, пересекающую $\bar{H}$ трансверсально в точке $x$. Пусть $\Gamma \subset \Pi$ - окружность малого радиуса с центром в $x$. Комплексная ориентация на $\mathbb{C P}^{2}$ определяет ориентацию на $\Gamma$. Пусть $\gamma-$ петля, состоящая из пути $L$ в $\mathbb{C P}^{2} \backslash \bar{H}$, соединяющего $p$ с точкой $q \in \Gamma$, окружности $\Gamma$ (с положительной ориентацией), имеющей начало и конец в $q$, и возврата в $p$ вдоль $L$ в обратном направлении. Такие петли $\gamma$ (и соответствующие им элементы в $\pi_{1}$ ) назьваются геометрическими образующими фундаментальной групшы $\pi_{1}=\pi_{1}\left(\mathbb{C P}^{2} \backslash \bar{H}, p\right)$. Хорошо известно, что $\pi_{1}$ порождается геометрическими образуюшими, и если $\bar{H}$ - неприводимая кривая, то любые две геометрические образуюшие сопряжены в $\pi_{1}$.

Для каждой особой точки $s_{i}$ кривой $\bar{H}$ кратности $k_{i}$ выберем малую окрестность $U_{i} \subset \mathbb{C P}^{2}$ такую, что $\bar{H} \cap U_{i}$ задается (в локальных координатах в $U_{i}$ ) нормализованным уравнением Вейерштрасса

$$
w^{k_{i}}+\sum_{j=0}^{k_{i}-1} c_{j}(z) w^{j}=0
$$


(в частности, $w^{2}=z^{3}$, если $s_{i}$ является обыкновенным каспом, и $w^{2}=z^{2}$, если $s_{i}-$ ноуд). Пусть $p_{i}-$ произвольная точка в $U_{i} \backslash \bar{H}$. Напомним, что если $s_{i}-$ обыкновенный касп, то $\pi_{1}\left(U_{i} \backslash \bar{H}, p_{i}\right)$ изоморфна группе кос $\mathrm{Br}_{3}$ из трех нитей и порождена двумя геометрическими образуюшими, скажем $a_{1}$ и $a_{2}$, удовлетворяющими соотношению $a_{1} a_{2} a_{1}=a_{2} a_{1} a_{2}$, и если $s_{i}-$ ноуд, то $\pi_{1}\left(U_{i} \backslash \bar{H}, p_{i}\right)$ изоморфна $\mathbb{Z} \oplus \mathbb{Z}$ и порождена двумя коммутируюшими геометрическими образуюшими.

Выберем гладкие пути $L_{i}$ в $\mathbb{C P}^{2} \backslash \bar{H}$, соединяюшие точки $p_{i}$ с точкой $p$. Этот выбор определяет гомоморфизмы $\psi_{i}: \pi_{1}\left(U_{i} \backslash \bar{H}, p_{i}\right) \rightarrow \pi_{1}$. Назовем группу $\psi_{i}\left(\pi_{1}\left(U_{i} \backslash \bar{H}, p_{i}\right)\right)=G_{i}$ локальной фундаментальной группой особой точки $s_{i}$. Локальные фундаментальные группы $G_{i}$ зависят от выбора путей $L_{i}$ и, следовательно, определены однозначно с точностью до сопряжения в $\pi_{1}$. Если брэйд-монодромное разложение $\operatorname{bmf}(\bar{H})$ равно $s=u_{1} \cdot \ldots \cdot u_{n} \in S_{\mathrm{Br}_{m}}$ и множитель $u_{i}$ соответствует особой точке $s_{i}$ кривой $\bar{H}$, то ввиду теоремы Зарискогован Кампена группа $G_{i}$ совпадает (с точностью до сопряжения) с образом группы

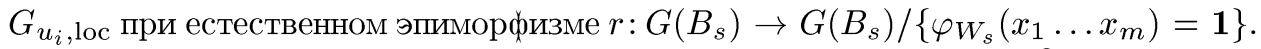
Монодромия $\bar{\mu}: \pi_{1} \rightarrow \mathfrak{S}_{N}$ обшего накрытия плоскости $f: X \rightarrow \mathbb{C P}^{2}$, разветвленного вдоль $\bar{H}$, определяет гомоморфизм $\mu=\bar{\mu} \circ r: G(s) \rightarrow \mathfrak{S}_{N}$, который также называется монодромией отображения $f$.

Конечный морфизм $f: X \rightarrow \mathbb{C P}^{2}, \operatorname{deg} f=N$, разветвленньй над каспидальной кривой $\bar{H}$, называется общим накрытием плоскости с дискриминантной кривой $\bar{H}$, если монодромия $\mu$ отображения $f$ является общим гомоморфизмом, т.е. удовлетворяет следуюшим условиям:

(i) $\mu$-эпиморфизм;

(ii) образ $\mu(\gamma)$ каждой геометрической образуюшей $\gamma$ является транспозицией в симметрической группе $\mathfrak{S}_{N}$;

(iii) образы $\mu\left(G_{i}\right)$ локальных фундаментальных групп $G_{i}$, соответствуюших особым точкам кривой $\bar{H}$, являются подгруппами в $\mathfrak{S}_{N}$ порядков больше чем 2 .

Последнее условие эквивалентно тому, что $X$ является неособой алгебраической поверхностью (см., например, [10]). Отметим, что если $\bar{H}$ является дискриминантной кривой некоторого обшего накрытия, то ее степень является четным числом. Для общего накрытия $f$ полный прообраз $f^{*}(\bar{H})$ распадается (если $\operatorname{deg} f>2$ ) в сумму: $f^{*}(\bar{H})=2 R+C$, где $R \subset X$ - дивизор ветвления отображения $f$, имеющий кратность ветвления, равную двум, и $C$ - приведенная кривая, в которой $f$ не разветвлено. Ограничение $f_{\mid R}$ на $R$ является морфизмом степени 1.

В симплектическом случае, как это было замечено М. Громовым, приведенное выше обсуждение свойств общих накрытий плоскости в терминах монодромий общих накрытий может быть перенесено также и в симплектическую ситуацию (см. [5, лемма 1] и $[2$, предложение 10]). Во-первых, если заданы каспидальная симплектическая поверхность $\bar{H}$ (возможно, с отрищательными ноудами) в $\mathbb{C P}^{2}$ и общий эпиморфизм (монодромия) $\bar{\mu}$ из фундаментальной групшы ее дополнения в симметрическую группу $\mathfrak{S}_{N}$, то можно построить гладкое накрытие $f: X \rightarrow \mathbb{C P}^{2}$ степени $N$, разветвленное над $\bar{H}$. Прообраз симплектической формы обрашается в нуль на нормальном расслоении к поверхности ветвления $R \subset X$ и в точках прообраза каспидальных точек поверхности $\bar{H}$, лежащих на $R$. Однако можно добавить точную 2 -форму $\eta \in \Lambda^{2}(X)$ так, что форма $f^{*} \omega_{\mathbb{C P}^{2}}+\eta$ станет симплектической. Сушественно здесь то, что хотя нет канонического подъема симплектической 
$\oint о р м ы$ на многообразие $X$, тем не менее, полученная в результате симплектическая структура определена канонически благодаря аргументам мозерова типа.

Сформулируем все сказанное выше в виде следующей леммы.

ЛЕммА 7.1. Предположим, что каспидальная симплектическая поверхность $\bar{H} \subset \mathbb{C P}^{2}$ (возможно, с отрицательньми ноудами) имеет общуло монодромию $\bar{\mu}: \pi_{1}\left(\mathbb{C P}^{2} \backslash \bar{H}\right) \rightarrow \mathfrak{S}_{n}$. Тогда существует единственное симплектическое четырехмерное многообразие $X$ с гладким отображением $f: X \rightarrow$ $\mathbb{C P}^{2}$, разветвленным над $\bar{H}$, как это описано выше, такое, что симплектическая структура на $X$ является поднятием стандартной симплектической структурьи на $\mathbb{C P}^{2}$.

Ясно, что если задана симплектическая изотопия $\bar{H}_{t}, t \in[0,1]$, каспидальных симплектических поверхностей и сушествует эпиморфизм $\bar{\mu}: \pi_{1}\left(\mathbb{C P}^{2} \backslash \bar{H}_{0}\right) \rightarrow$ $\mathfrak{S}_{N}$, то мы получим семейство симплектических четырехмерных многообразий $X_{t}$. (Напомним, что по определению изотопии топология дополнения $\mathbb{C P}^{2} \backslash \bar{H}_{t}$ не меняется.) Используя аргументы, аналогичные применявшимся в [7], можно показать, что любые симплектические поверхности с отрицательными ноудами в $\mathbb{C P}^{2}$ симплектически изотопны каспидальным симплектическим кривым Гурвица с отрицательными ноудами. Поэтому в симплектическом случае при исследовании общих накрытий плоскости $\mathbb{C P}^{2}$, разветвленных вдоль каспидальных симплектических поверхностей $\bar{H}$, можно ограничиться случаем, когда $\bar{H}$ является кривой Гурвища.

Рассмотрим два обших накрытия $f^{\prime}: X^{\prime} \rightarrow \mathbb{C P}^{2}$ и $f^{\prime \prime}: X^{\prime \prime} \rightarrow \mathbb{C P}^{2}$ степени $N$, разветвленных соответственно вдоль каспидальных кривых Гурвица $\overline{H^{\prime}}$ и $\bar{H}^{\prime \prime}$ (возможно, с отрицательными ноудами). Пусть $s^{\prime}=\operatorname{bmf}\left(\bar{H}^{\prime}\right)$ и $s^{\prime \prime}=\operatorname{bmf}\left(\bar{H}^{\prime \prime}\right)$ - разложения на множители брэйд-монодромии кривых $\bar{H}^{\prime}$ и $\bar{H}^{\prime \prime}$, накрытие $f^{\prime}$ (соответственно, $\left.f^{\prime \prime}\right)$ определено монодромией $\mu^{\prime}: G\left(s^{\prime}\right) \rightarrow \mathfrak{S}_{N}$ (соответственно, $\left.\mu^{\prime \prime}\right)$. Скажем, что $\bar{H}^{\prime}$ и $\bar{H}^{\prime \prime}$ являются слабо $\mu$-эквивалентнылми, если пары $\left(s^{\prime}, \mu_{s^{\prime}}\right)$ и $\left(s^{\prime \prime}, \mu_{s^{\prime \prime}}\right)$ слабо $\mu$-эквивалентны.

Лемма 7.2. Пусть две кривые Гурвица $\bar{H}^{\prime}$ и $\bar{H}^{\prime \prime}$ являются кривыми ветвления двух общих накрытий $f^{\prime} u f^{\prime \prime}$ плоскости. Если $\bar{H}^{\prime}$ и $\bar{H}^{\prime \prime}$ слабо лентны, то существует симплектоморфизм $h: X^{\prime} \rightarrow X^{\prime \prime}$ такой, что накрьтия $f^{\prime} u f^{\prime \prime} \circ h$ являются изотопнымии.

ДоКАЗАТЕЛЬСТво следует из определения слабой $\mu$-эквивалентности, утверждения 5.5 и теоремы 3.3 , доказанной в [7].

В алгебраическом случае, если $(X, L)$ - поляризованная проективная поверхность, где $L$ - очень обильное линейное расслоение на $X$, для достаточно большого $k$ сечения расслоения $L^{\otimes k}$ определяют вложение поверхности $X$ в некоторое проективное пространство $\mathbb{C P}^{r}$. Ограничение общей проекции $\mathbb{C P}^{r} \rightarrow \mathbb{C P}^{2}$ на $X$ дает общее накрытие $f_{k}: X \rightarrow \mathbb{C P}^{2}$, разветвленное в некоторой кривой $\bar{H}_{k}$. Заметим, что степень накрытия равна $k^{2} \operatorname{deg} L=k^{2} c_{1}(L)^{2}$. Пространство общих проекций является связным, следовательно, корректно следующее определение.

ОПРЕДЕЛЕНИЕ 7.3 . Назовем $k$-м брәйд-монодромным инвариантом $\mu_{k}(X, L)$ поляризованной комплексной поверхности $(X, L)$ тип брэйд-монодромного разложения на множители кривой $\bar{H}_{k}$. 
Поляризация определяет канонически симплектическую структуру $\omega_{L}$ на $X$. Для ее определения выберем положительную $(1,1)$-форму, представляющую первый класс Черна $c_{1}(L) \in H^{2}(X, \mathbb{Z})$. Любые две такие формы можно соединить линейной гомотопией, следовательно, они диффеоморфны по теореме Мозера.

ТЕОРЕМа 7.4. Пусть $(X, L) u\left(X^{\prime}, L^{\prime}\right)$ - две поляризованные проективные поверхности. Предполохсим, что $\mu_{k}(X, L)=\mu_{k}\left(X^{\prime}, L^{\prime}\right)$ для некоторого $k$ такого, что $k^{2} c_{1}(L)^{2} \geqslant 12$. Тогда $\left(X, \omega_{L}\right) u\left(X^{\prime}, \omega_{L^{\prime}}\right)$ симплектоморфнь.

ДоКАЗАТЕЛЬСТВО. По предположению кривые ветвления $\bar{H}$ и $\bar{H}^{\prime}$ общих проекций на $\mathbb{C P}^{2}$, определенных соответственно сечениями расслоений $L^{\otimes k}$ и $\left(L^{\prime}\right)^{\otimes k}$, имеют один и тот же тип разложений брэйд-монодромии на множители. Таким образом, согласно [7, теорема 3.3$]$ кривые $\bar{H}$ и $\bar{H}^{\prime}$ являются симплектически изотопными как каспидальные кривые Гурвица. Из [12, теорема 3] следует, что существует единственная общая монодромия $\mu: \pi_{1}\left(\mathbb{C P}^{2} \backslash \bar{H}\right) \rightarrow \mathfrak{S}_{N}$ для $N=k^{2} c_{1}\left(L_{1}\right)^{2}$. Следовательно, ввиду леммы 7.1 имеем единственное семейство разветвленных симплектических накрытий плоскости $\mathbb{C P}^{2}$, связывающее многообразия $X$ и $X^{\prime}$. Из теоремы Мозера следует, что $X$ и $X^{\prime}$ симплектоморфны относительно поднятых с плоскости симплектических структур. С другой стороны, эти поднятые структуры пропорциональны с одним и тем же коэффициентом $k$ симплектическим структурам, определенньм поляризациями, что завершает доказательство теоремы.

Пусть $(X, \omega)$ - компактное симплектическое четырехмерное многообразие с симплектической формой $\omega$, класс когомологий которой $[\omega] \in H^{2}(X, \mathbb{Z})$. Зафиксируем совместимую с $\omega$ почти комплексную структуру $J$ и соответствующую риманову метрику $g$. Пусть $L$ - линейное расслоение на $X$, первый класс Черна которого есть $[\omega]$. В [2] доказано, что для $k \gg 0$ линейное расслоение $L^{\otimes k}$ имеет много почти голоморфных сечений, так что можно выбрать три из них, которые дают почти голоморфное общее накрытие $f_{k}: X \rightarrow \mathbb{C P}^{2}$ степени $N_{k}=k^{2} \omega^{2}$, разветвленное вдоль каспидальной кривой Гурвица $\bar{H}$ (возможно, с отрицательными ноудами). Обозначим, как и выше, через $\operatorname{bmf}\left(\bar{H}_{k}\right)=s_{k}$ разложение на множители брэйд-монодромии кривой Гурвища $\bar{H}_{k}$ и через $\mu_{k}: G\left(B_{s_{k}}\right) \rightarrow \mathfrak{S}_{N_{k}}-$ монодромию накрытия $f_{k}$.

ОПРЕДЕЛЕниЕ 7.5. Пара $\left(\mathrm{bmf}\left(\bar{H}_{k}\right), \mu_{k}\right)$ называется $k$-м брәйд-монодромнымм инвариантом $\bar{\mu}_{k}(X, \omega)$ симплектического четырехмерного многообразия $(X, \omega)$.

ТЕОРема 7.6 [3]. Если два симплектических четырехмерных многообразия $\left(X^{\prime}, \omega^{\prime}\right)$ и $\left(X^{\prime \prime}, \omega^{\prime \prime}\right)$ симплектоморфнь, то для достаточно большого $k$ брэйд-монодромные инварианть $\mu_{k}\left(X^{\prime}, \omega^{\prime}\right) u \mu_{k}\left(X^{\prime \prime}, \omega^{\prime \prime}\right)$ являются слабо $\mu$-эквивалентными.

Появление условия слабой эквивалентности в теореме 7.6 связано с конструкцией изотопии накрытий, которая непрерывно зависит от выбора ручных почти комплексных структур. Поэтому для симплектических четырехмерных многообразий мы получаем "регулярную гомотопию" кривых ветвления, которая приводит к слабой $\mu$-эквивалентности их брэйд-монодромных разложений на множители. Из следующей теоремы вытекает, что в общем случае мы не можем избежать появления отрицательных ноудов. 
ТЕОРемА 7.7. Для любого $N \geqslant 4$ существуют два регулярно гомотопных общих накрытия $f_{1}: X \rightarrow \mathbb{C P}^{2}$ и $f_{2}: X \rightarrow \mathbb{C P}^{2}$ степени $N$, разветвленных вдоль каспидальных кривых Гурвица $\bar{H}_{1}$ и $\bar{H}_{2}$ без отричательных ноудов, такие, что:

(i) $\bar{H}_{1}$ и $\bar{H}_{2}$ имеют разные типь брәйд-монодромных разложений на множители;

(ii) пары $\left(\operatorname{bmf}\left(\bar{H}_{1}\right), \mu_{1}\right) u\left(\operatorname{bmf}\left(\bar{H}_{2}\right), \mu_{2}\right)$ слабо $\mu$-эквивалентны.

ДокАЗАТЕЛЬСТВо. Пусть $\bar{H}_{N}$ - дискриминантная кривая общей линейной проекции pr: $X_{N} \rightarrow \mathbb{C P}^{2}$ гладкой проективной поверхности $X_{N} \subset \mathbb{C P}^{3}$ степени $\operatorname{deg} X_{N}=N$. Обозначим через $m=N(N-1)$ степень кривой $\bar{H}_{N}$ и через $s=s_{N}=\operatorname{bmf}\left(\bar{H}_{N}\right)-$ разложение на множители ее брэйд-монодромии. Проекция pr определяет обший эпиморфизм (монодромию) $\mu=\mu_{N}: G\left(B_{s}\right) \rightarrow \mathfrak{S}_{N}$. В работе [14] доказано, что $C$-группа

$$
G(s)=G\left(W\left(B_{s}, \mathbb{F}_{m}\right)\right) \simeq\left\langle x_{1}, \ldots, x_{m} \mid x_{i}^{-1} b\left(x_{i}\right)=\mathbf{1}, i=1, \ldots, m, b \in B_{s}\right\rangle
$$

$C$-изоморфна группе кос $\mathrm{Br}_{N}$ и $\mu$ совпадает с каноническим эпиморфизмом $\sigma$ : $\operatorname{Br}_{N} \rightarrow \mathfrak{S}_{N}$, имеюшим своим ядром группу крашеных кос. Без ограничения общности можно предполагать, что $\varphi_{s}\left(x_{1}\right)=a_{1} \in \mathrm{Br}_{N}$. Поскольку $\mu$ является эпиморфизмом, то сушествует элемент $y$, сопряженньй в $\mathbb{F} m$ некоторой образующей $x_{i}$ и такой, что $\varphi_{s}(y)=a_{2}^{-2} a_{3} a_{2}^{2}$. Заметим, что $\left[\varphi_{s}\left(x_{1}\right), \varphi_{s}(y)\right] \neq \mathbf{1}$ в $\operatorname{Br}_{N}$, но $\mu\left(\varphi_{s}\left(x_{1}\right)\right)=(1,2)$ и $\mu\left(\varphi_{s}(y)\right)=(3,4)$ являются коммутируюшими транспозищиями в группе $\mathfrak{S}_{N}$. Тогда из теоремы 4.2 следует, что существуют число $M \in \mathbb{N}$ и две пары $\left(\bar{s}, \mu_{\bar{s}}\right)$ и $\left(\tilde{s}, \mu_{\tilde{s}}\right), \bar{s}, \tilde{s} \in \mathscr{A}_{2} \subset S_{\mathrm{Br}_{M}}$, такие, что:

1) $\alpha(\bar{s})=\alpha(\tilde{s})=\Delta_{M}^{2}$

2) $G_{\bar{s}}$ является $C$-изоморфной $C$-группе $G(s) \simeq \mathrm{Br}_{N}$, a $G_{\tilde{s}}$ является $C$-изоморфной $C$-группе $\mathrm{Br}_{N} /\left\{\left[a_{1}, a_{2}^{-2} a_{3} a_{2}^{2}\right]=\mathbf{1}\right\}$;

3) элементы $\bar{s}$ и $\tilde{s}$ имеют различные типы;

4) $\mu_{\bar{s}}$ и $\mu_{\tilde{s}}$ являются общими эпиморфизмами в группу $\mathfrak{S}_{N}$;

$5)$ пары $\left(\bar{s}, \mu_{\bar{s}}\right)$ и $\left(\tilde{s}, \mu_{\tilde{s}}\right)$ слабо $\mu$-эквивалентны;

6) $\bar{s}, \tilde{s} \in \mathscr{A}_{2}^{0} \subset S_{\mathrm{Br}_{M}}$.

Согласно теореме 5.3 сушествуют две каспидальные кривые Гурвища $\bar{H}_{1}$ и $\bar{H}_{2}$ в $\mathbb{C P}^{2}$ степени $M$, имеюшие $\operatorname{bmf}\left(\bar{H}_{1}\right)=\bar{s}$ и $\operatorname{bmf}\left(\bar{H}_{2}\right)=\tilde{s}$. Общие эпиморфизмы $\mu_{\bar{s}}$ и $\mu_{\tilde{s}}$ определяют два общих накрытия $f^{\prime}: X^{\prime} \rightarrow \mathbb{C P}^{2}$ и $f^{\prime \prime}: X^{\prime \prime} \rightarrow \mathbb{C P}^{2}$, разветвленные соответственно вдоль $\bar{H}_{1}$ и $\bar{H}_{2}$. Из леммы 7.2 следует, что существует симплектоморфизм $h: X^{\prime} \rightarrow X^{\prime \prime}$ такой, что накрытия $f_{1}=f^{\prime}: X=X^{\prime} \rightarrow \mathbb{C P}^{2}$ и $f_{2}=f^{\prime \prime} \circ h: X \rightarrow \mathbb{C P}^{2}$ являются регулярно гомотопными, но их гурвищовские кривые ветвления имеют разные типы разложений на множители брэйд-монодромии.

\section{Список литературы}

1. Artin E. Theorie der Zopfe // Hamburg Abh. 1925. V. 4. P. 47-72.

2. Auroux D. Symplectic 4-manifolds as branched coverings of $\mathbb{P}^{2} / /$ Invent. Math. 2000. V. 139. P. 551-602.

3. Auroux D., Katzarkov L. Branched coverings of $\mathbb{P}^{2}$ and invariants of symplectic 4-manifolds // Invent. Math. 2000. V. 142. P. 631-673.

4. Burde G., Zieschang H. Knots. Berlin-N. Y.: Walter de Gruyter, 1985. 
5. Gompf $R$. Symplectically aspherical manifolds with nontrivial $\pi_{2} / /$ Math. Res. Letters. 1998. V. 5. P. 599-603.

6. Grauert H., Remmert R. Komplexe Raume // Math. Ann. 1958. V. 136. P. 245-318.

7. Куликов Вик. С., Харламов В.M. О брэйд-монодромных разложениях на множители // Изв. РАН. Сер. матем. 2003. Т. 67. № 3. С. 79-118.

8. Куликов B. C., Куликов Buк. C. Общие накрытия плоскости с $A-D$-E-особенностями // Изв. РАН. Сер. матем. 2000. Т. 64. №6. С. 1153-1195.

9. Куликов Вик. C., Тайхер M. Брэйд-монодромные разложения на множители и диффеоморфные типы // Изв. РАН. Сер. матем. 2000. Т. 64. № 2. С. 311-341.

10. Куликов Вик. С. О гипотезе Кизини // Изв. РАН. Сер. матем. 1999. Т. 63 . №6. C. $1139-1170$.

11. Куликов Вик. C. Геометрическая реализация $C$-групп // Изв. РАН. Сер. матем. 1995. T. 45. №1. C. 197-206.

12. Куликов Вик. С. Обобщенная гипотеза Кизини // Тр. МИАН. 2003. Т. 241. С. 122-131.

13. Немировский С. Ю. К теореме Куликова о гипотезе Кизини // Изв. РАН. Сер. матем. 2001. T. 65. № 1. C. $71-74$.

14. Moishezon B. Stable branch curves and braid monodromies // Lect. Notes Math. 1981. V. 862. P. 107-192.

15. Moishezon B. The arithmetic of braids and a statement of Chisini // Contemp. Math. 1994. V. 164. P. 151-175.

16. Moishezon B., Teicher $M$. Braid group technique in complex geometry. I. Line arrangements in $\mathbb{C P}^{2}$ // Contemp. Math. 1988. V. 78. P. 425-555.

17. Moishezon B., Teicher $M$. Braid group technique in complex geometry. V. The fundamental group of a complement of a branch curve of a Veronese generic projection // Comm. Anal. Geom. 1996. V. 4. № 11. P. 1-120.

Математический институт

Поступило в редакцию

им. В.А. Стеклова РАН

26.08.2003

E-mail: kulikov@mi.ras.ru 